\title{
Bounded Relative Orbits in the Zonal Problem via High-Order Poincaré Maps
}

\author{
Yanchao He * \\ Beihang University, 100191 Beijing, People's Republic of China \\ Roberto Armellin ${ }^{\dagger}$ \\ University of Surrey, Guildford, Surrey, GU2 7XH, United Kingdom \\ Ming $\mathrm{Xu}$ * \\ Beihang University, 100191 Beijing, People's Republic of China
}

\begin{abstract}
Searching for naturally bounded relative orbits in a zonal gravitational field is a crucial and challenging task in astrodynamics. In this work, a semi-analytical approach based on high-order Taylor expansions of Poincaré maps is developed. Entire families of periodic orbits, parameterized by the energy and the polar component of the angular momentum, are computed under arbitrary order zonal harmonic perturbations, thus enabling the straightforward design of missions with prescribed properties. The same technique is then proven effective in determining quasi-periodic orbits that are in bounded relative motion for long time and with very large aperture. Finally, an illustrative example on how to frame the design of bounded relative orbits with prescribed properties as an optimization problem is presented.
\end{abstract}

\section{Introduction}

$\mathrm{F}$ ORMATION flying is an emerging technology that features the collaboration of two or more spacecraft to achieve shared mission objectives. The formation flying concept can overcome some of the limitations associated with a single-spacecraft mission. The benefits that can be obtained by distributing a payload over multiple platforms includes simpler designs, cheaper replacement resulting in higher redundancy, realization of larger baselines, and the ability to observe targets from multiple angles or at multiple times. Because of this, formation flying has developed into one of the most stirring space technologies. The concept has already been successfully tested in orbit with missions such as PRISMA [1], GRACE [2], and TerraSAR-X (TSX) \& TanDEM-X (TDX) [3], which have further stimulated the research by astronautical scholars and organizations.

The design of configurations that enables spacecraft to fly in formation both satisfying constraints on relative distances and minimizing stationkeeping maneuvers is of fundamental importance to either reduce the launch mass and overall mission costs or to increase mission lifetime and scientific/economic return. To achieve this goal it is necessary

*PhD Student, School of Astronautics; heyanchao@buaa.edu.cn.

${ }^{\dagger}$ Senior Lecturer, Surrey Space Centre, BA Building; r.armellin@surrey.ac.uk.

$¥$ Associate Professor, School of Astronautics; xuming@buaa.edu.cn (corresponding author). 
to study the spacecraft relative dynamics and determine the initial conditions that generate bounded relative orbits. When Keplerian dynamics are considered, bounded relative motion can be achieved either by finding appropriate initial conditions that produce periodic relative orbits in the linearized relative equations of motion (Hill-Clohessy-Wiltshire [4, 5] and Tschauner-Hempel equations [6]) or by matching the energy between neighboring orbits in the full nonlinear relative dynamics [7]. The solutions in the two-body approximation provide good insight into the relative dynamics problem; however, when applied in real-word scenarios, expensive formation-keeping strategies are required to cope with the effect of unmodeled perturbations [8-11].

A strategy to limit the formation maintenance costs consists in designing bounded relative motion in a relative dynamical model that includes as many perturbations as possible. In this context, astrodynamicists posed their attention on the identification of bounded relative orbits under zonal harmonic perturbations, mainly $J_{2}$. Schaub and Alfriend [12] were the first to propose first-order conditions that enable $J_{2}$-invariant relative orbits. These conditions were obtained by setting the chief and deputy on orbits with equal rate of both the right ascension of the ascending node (RAAN) and mean argument of latitude [12]. It is important to note that in this work mean orbital elements were considered, thus the effect of short-periodic oscillations was neglected. Due to this approximation and the error introduced by linearization, the relative motion remains bounded only for short times and small relative distances. Later studies aimed to overcome these limitations. In [13] more general conditions for $J_{2}$-invariant relative orbits were obtained by using non-osculating orbital elements based on the concept of gauge-generalized equations. Dang et al. [14] introduced an additional corrective factor to the standard invariant constraints to enhance the performance of $J_{2}$ invariance. The first-order bounded constraints under higher-order $\left(J_{n}, n \geq 2\right)$ zonal perturbations were given in [15] by initializing the chief on the frozen orbit. Despite the fact that mean orbital elements-based analytical approaches are limited in the size and duration of the bounded relative orbits, they offer a clear mathematical and physical insight into the nature of bounded relative motion generation problem.

Within the framework of analytical theory for bounded relative motion in the $J_{2}$ problem, the investigations performed by Lara and Gurfil [16] and Chu et al. [17] are worth being mentioned. In the first work a radial intermediary Hamiltonian was introduced to obtain an integrable approximation of $J_{2}$-perturbed relative motion without averaging the short-periodic terms. By imposing the relative motion boundedness conditions from [18, 19], numerical simulations showed that the intermediary-based orbits could produce bounded relative motion in the full $J_{2}$ perturbation at various inclinations. Nevertheless, only the argument of the perigee and RAAN were left as degrees of freedom, whereas the chief and deputy must have the same values of semi-major axis, eccentricity, inclination, and true anomaly. The second work was based on another approximation of the gravitational potential and presented a full separable Hamiltonian in terms of spherical coordinates. By applying the Hamilton-Jacobi theory and action-angle variables, canonical solutions were derived resulting in both constant nodal period $\left(T_{d}\right)$ and drift of RAAN per nodal period $\left(\Delta \Omega_{d}\right)$ for the pseudo-elliptical orbits. Thus, matching these two constant values between pair of orbits achieved the bounded relative 
motion.

In parallel to the analytical studies, several numerical methods were proposed to tackle the problem. Koon et al. [20] introduced the Routh reduction and Poincaré section techniques, originally devised by Broucke [21], for the investigation of periodic orbits in the $J_{2}$ problem. However, as first observed by Xu et al. [22] and subsequently discussed by Baresi and Scheeres [23, 24], the initialization of the chief on a pseudo-circular (periodic) orbit and deputies on pseudo-elliptical (quasi-periodic) orbits with the same energy level is not suitable for the generation of bounded relative motion. To illustrate the reason, Xu et al. [22] studied the mapping from $\left(E, H_{z}, \Delta r\right)$ to $\left(T_{d}, \Delta \Omega_{d}\right)$, where $E$ is the Jacobi energy, $H_{z}$ is the polar component of the angular momentum and $\Delta r$ is the measure of distance between the pseudo-circular orbit and its pseudo-elliptical orbits. Using a fully numerical approach it was shown that for $\Delta r \neq 0$ the same value of image $\left(T_{d}, \Delta \Omega_{d}\right)$ corresponds to different values of inverse image $\left(E, H_{z}\right)$, thus demonstrating the existence of bounded relative orbits. Further and comprehensively, Baresi and Scheeres [23] obtained entire families of quasi-periodic orbits producing bounded relative motion about a periodic one. This result was achieved via stroboscopic maps and a fully numerical technique, referred to as revised GMOS algorithm [25]. In the same work the authors showed the validity of the algorithm applied to the primary of the binary asteroid $1999 \mathrm{KW} 4 \alpha$, under $J_{5}$ zonal harmonic perturbations. In [24] the same revised GMOS method was used to study the bounded relative motion at different altitudes (low-Earth orbit, medium-Earth-orbit, and geostationary-Earth-orbit) and for scenarios with either mission-specified or user-defined $T_{d}$. Unlike the previous results computed with analytical methods, the aforementioned numerical ones were developed to satisfy the bounded relative motion conditions without any approximation. Consequently, mission designers can utilize the provided initial conditions to initialize the neighboring orbits yielding bounded relative orbits with arbitrary size over very long periods of time (or infinite time in theory). However, the achievement of such highly accurate results requires the implementation of a complex algorithm that deals with the numerical propagation of the dynamics and its associated variational equations for several initial conditions, followed by the solution of a large-scale system of nonlinear equations through Newton's iterations [23, 24].

The aim of this work is to introduce semi-analytical techniques for the design of bounded relative motion in the Earth's zonal problem. The idea is based on the work by Wittig and Armellin [26], in which it was shown that the computation of high-order Taylor expansions of the flow of repetitive systems enabled the forward propagation of a point for many revolutions by the repetitive evaluation of a Taylor map. As in [26], the high-order Taylor polynomials computed in this work are obtained via differential algebra (DA) techniques. These were first introduced in the field of particle accelerator physics by Berz [27] and later applied in a variety of problems in astrodynamics, including asteroid encounter analysis [28], uncertainty propagation [29], orbital conjunctions assessment [30], space debris evolution [31], and initial orbit determination [32]. In this work the key capability of DA to efficiently expand both the flow of ordinary differential equations (ODEs) and the solution of parametric implicit equations (PIEs) is exploited to compute the high-order Taylor expansion of Poincaré maps with respect to both initial conditions and an arbitrary number of relevant 
parameters. This feature is first used to compute families of periodic orbits (in the $J_{15}$ zonal problem) parametrized in both $E$ and $H_{z}$. These two degrees of freedom are then used to design a periodic orbit with specified properties, e.g. repeat ground-track (RGT) and sun-synchronous (SS). The expansion of Poincaré map about the selected fixed point then allows propagating neighboring conditions for thousands of revolutions by simple evaluations of a Taylor map, similarly to what shown in [26]. The degrees of freedom available in the Poincaré map are exploited to compute families of quasi-periodic orbits with desired properties. In particular, families of quasi-periodic orbits in bounded relative motion are obtained by setting matching conditions on $T_{d}$ and $\Delta \Omega_{d}$. The achieved relative orbits are bounded for very long times and for aperture size comparable with fully numerical methods, with the advantage that the entire set of initial conditions producing bounded relative motion is obtained via a single orbit propagation for a single nodal period.

In order to explain our DA-based semi-analytical approach, the remainder of this paper is presented as follows. In Sec. II. we use the Routh's reduction approach to model the orbital dynamics in the zonal gravitational field. This is followed by a brief introduction of the DA techniques and their use for the high-order expansion of the solution of ODEs and PIEs (Sec.III). In Sec.IV] fixed points (i.e., periodic orbits or quasi-circular orbits [21]) are computed, and the family of fixed points parametrized by $E$ and $H_{z}$ is also obtained only by the analytical evaluation of Taylor polynomials. Furthermore, the effects the zonal harmonic order on the constrained fixed points are also discussed to illustrate the convergence of the results for order greater than ten. In Sec. $\mathrm{V}$, a systematic DA-based method combined with the high-order Poincaré maps is proposed to compute the central manifolds (i.e., quasi-periodic orbits or quasi-elliptical orbits [21]) around the fixed point (away from the critical inclination case). This result enables the identification of the family of bounded relative orbits for different values of $H_{z}$ and $E$. Finally, an example on how to design the bounded relative orbits with predefined targets on maximum radial and cross-track separation is provided. Conclusions are drawn in Sec.VI]

\section{Reduced Orbital Dynamics of the Zonal Problem}

In the inertial frame of an axial symmetric celestial body with the origin located at its center, the $x$ axis pointing to the vernal equinox, the $z$ axis along the body's pole direction and the $y$ axis completing the orthogonal triad, the zonal gravitational potential is generally modeled as

$$
V=-\frac{\mu}{\rho}+\frac{\mu}{\rho} \sum_{n=2}^{\infty} J_{n}\left(\frac{R}{\rho}\right)^{n} P_{n}(\sin \alpha)
$$

in which $\mu$ is the gravitational parameter of the central body, $\rho$ is the distance of the spacecraft from the mass center of the body, $R$ is the mean radius of the body, $J_{n}(n \geq 2)$ is the zonal harmonic coefficient of degree $n, \alpha$ is the latitude of the spacecraft, and $P_{n}(\sin \alpha)$ are the latitude-associated Legendre polynomials. The degree $n$ needs to be selected according to the required accuracy. As an example, in the work by He et al. [33], it was pointed out that $n=15$ provided 
sufficiently stable values of the nodal period for spacecraft orbiting the Earth. Unless otherwise indicated, all the parameters and values reported in this paper are scaled by the characteristic length $R=6378.1363 \mathrm{~km}$ and characteristic time $\sqrt{R^{3} / \mu}=806.81099 \mathrm{sec}$.

As shown in the previous research from [20-24,34], using Routh reduction and introducing the cylindrical coordinate variables $(r, z, \phi)$ with the coordinate transformation

$$
\left\{\begin{array}{l}
\sin \varphi=z / \rho, \\
\rho=\sqrt{r^{2}+z^{2}}
\end{array}\right.
$$

the $J_{n}$ Hamiltonian governing the motion is expressed by

$$
\mathcal{H}(r, z, \dot{r}, \dot{z})=\frac{1}{2}\left(\dot{r}^{2}+\dot{z}^{2}+\frac{H_{z}^{2}}{r^{2}}\right)+V(r, z)
$$

in which the integral of motion $H_{z}=r^{2} \dot{\phi}$ is the polar component of the angular momentum, $(\dot{r}, \dot{z}, \dot{\phi})$ are the conjugate momenta of variables $(r, z, \phi)$. The variables $(r, z, \phi)$ represent the distance from the spacecraft to the $z$ axis, the height above or below the equatorial plane, and the instantaneous longitude of meridian plane of the spacecraft, respectively. As the $J_{n}$ Hamiltonian [Eq. [3] ] is time-independent, it is an integral of motion and it coincides with the energy $E$ of the system. As in previous studies, the pair $\left(E, H_{z}\right)$ is also used in our work to parameterize the orbits.

The time evolution of the spacecraft reduced state $x=[r, z, \dot{r}, \dot{z}]^{T}$ is determined in the zonal problem by the integration of the system of ODEs

$$
\dot{\boldsymbol{x}}=f\left(\boldsymbol{x}, H_{z}\right)=\left[\begin{array}{c}
\dot{r} \\
\dot{z} \\
\frac{H_{z}^{2}}{r^{3}}-\frac{\partial V}{\partial r} \\
-\frac{\partial V}{\partial z}
\end{array}\right] .
$$

Once the reduced state is integrated, the time derivative of the longitude is computed by $\dot{\phi}=H_{z} / r^{2}$ and the longitude $\phi(t)$ by the integral

$$
\phi(t)=\phi_{0}+H_{z} \int_{t_{0}}^{t} \frac{1}{r^{2}(\tau)} \mathrm{d} \tau
$$

in which $\phi_{0}$ is its initial value at time $t_{0}$. 
The six-dimensional Cartesian state of the spacecraft $\boldsymbol{y}=[x, y, z, \dot{x}, \dot{y}, \dot{z}]^{T}$ is determined via the transformation:

$$
\left\{\begin{aligned}
x(t) & =r(t) \cos \phi(t), \\
y(t) & =r(t) \sin \phi(t), \\
z(t) & =z(t), \\
\dot{x}(t) & =\dot{r}(t) \cos \phi(t)-r(t) \dot{\phi}(t) \sin \phi(t), \\
\dot{y}(t) & =\dot{r}(t) \sin \phi(t)+r(t) \dot{\phi}(t) \cos \phi(t), \\
\dot{z}(t) & =\dot{z}(t)
\end{aligned}\right.
$$

Finally, two relevant quantities for bounded relative motion design are the nodal period $T_{d}$ and the drift of the RAAN in one nodal period, $\Delta \Omega_{d}$. The first one is defined as the time interval between any two consecutive passages of the spacecraft at the ascending node. The latter can be computed from the spacecraft longitude by

$$
\Delta \Omega_{d}=\phi\left(t_{0}+T_{d}\right)-\phi_{0}-2 \pi \operatorname{sgn}\left(H_{z}\right)=\Delta \phi-2 \pi \operatorname{sgn}\left(H_{z}\right)
$$

in which $\operatorname{sgn}(*)$ indicates the sign function.

\section{Differential Algebra Techniques}

In this section we give a brief introduction to the DA framework and its application to the automatic computation of high-order expansions of the solution of ODEs and PIEs. The interested reader can find further details in the works of Berz [27] and Valli et al. [29].

\section{A. The Framework of Differential algebra}

DA enables the efficient tools to compute the derivatives of functions within a computerized environment. More specifically, by substituting the classical implementation of real algebra with the implementation of a new algebra of Taylor polynomials, any deterministic function $f$ of $v$ variables that is $C^{k+1}$ in the domain of interest $[-1,1]^{v}$ (these properties are assumed to hold for any function dealt with in this work) is expanded into its Taylor polynomial up to an arbitrary order $k$. In addition to basic algebraic operations, operations for differentiation and integration can be easily introduced in the algebra, thus finalizing the definition of the differential algebraic structure of DA. Similarly to algorithms for floating point arithmetic, various algorithms were introduced in DA, including methods to perform composition of functions, to invert them, to solve nonlinear systems explicitly, and to treat common elementary functions [27]. The DA used for the computations in this work was implemented by Dinamica [35] in the software DA Computational Engine (DACE), including all core DA functionality and a C++ interface. Before introducing DA-based 
algorithms, it is worth noting that time complexity of DA calculations is exponential with both $v$ and $k$. For applications in astrodynamics that require a large number of operations in 6 variables (e.g. the expansion of the flow of complex ODEs) it has been found that an expansion order of 6 represents, in most cases, a good compromise between accuracy of the Taylor expansion and computational time (see [29] for more details).

\section{B. High-Order Expansion of the Flow of an ODE}

An important application of DA is the automatic computation of the high-order Taylor expansion of the solution of ODEs with respect to either the initial conditions and/or any parameter of the dynamics [29, 36]. This can be achieved by replacing the classical floating point operations of the numerical integration scheme, including the evaluation of the right-hand side of the ODE, with the corresponding DA-based operations. In this way, starting from the DA representation of the initial condition $x_{0}$, the DA-based ODE integration supplies the Taylor expansion of the flow in $x_{0}$ at all the integration stpng, up to any final time $t_{f}$. Any explicit ODE integration scheme can be adapted to work in the DA framework in a straightforward way. For the numerical integrations presented in this paper, a DA version of a 7/8 Dormand-Prince (8-th order solution for propagation, 7-th order solution for step size control) Runge-Kutta scheme is used. The main advantage of the DA-based approach is that there is no need to write and integrate variational equations to obtain high-order expansions of the flow. It is therefore independent on the particular right hand side of the ODE and the method is quite efficient in terms of computational cost.

\section{Expansion of the Solution of Parametric Implicit Equations}

Well-established numerical techniques (e.g., Newton's method) exist to compute numerically the solution of an implicit equation

$$
c(x)=0,
$$

with $\boldsymbol{c}: \mathbb{R}^{n} \rightarrow \mathbb{R}^{n}$. Suppose the vector function $\boldsymbol{c}$ depends explicitly on a vector of parameters $\boldsymbol{p}$, which yields the PIE

$$
c(\boldsymbol{x}, \boldsymbol{p})=0 .
$$

The solution of Eq. (9) is the function $\boldsymbol{x}=\boldsymbol{f}(\boldsymbol{p})$ that solves the equality for any value of $\boldsymbol{p}$.

DA techniques can effectively solve the previous problem by representing $\boldsymbol{f}(\boldsymbol{p})$ in terms of its Taylor expansion with respect to $\boldsymbol{p}$. This result is achieved by applying partial inversion techniques as detailed in the work by Lizia et al. [36]. The final result is

$$
\boldsymbol{x}=\mathcal{T}_{\boldsymbol{f}}^{k}(\boldsymbol{p}),
$$

which is the $k$-th order Taylor expansion of the solution of the implicit equation. For every value of $\boldsymbol{p}$, the approximate 
solution of $\boldsymbol{c}(\boldsymbol{x}, \boldsymbol{p})=0$ can be easily computed by evaluating the Taylor polynomial [10]. Apparently, the solution obtained by means of the polynomial map (10) is a Taylor approximation of the exact solution of Eq. (9). The accuracy of the approximation depends on both the order of the Taylor expansion and the displacement of $\boldsymbol{x}$ from its reference value.

The capability of expanding the solution of PIEs is of key importance for this work, as it appears in the high-order expansion of Poincaré sections, the expansion of the quasi-circular orbits with respect to $E$ and $H_{z}$, and the computation of quasi-circular orbit with prescribed properties (e.g., RGT and SS).

\section{Families of Fixed Points in the Earth's Zonal Problem}

In this section the DA-based approach to compute families of fixed points (quasi-circular orbits) in the Earth's zonal problem is presented. Section IVA contains a general algorithm to compute the quasi-circular orbits, while in Sec. IVB we explain how to use DA tools to parametrize the fixed points with respect to $E$ and $H_{z}$. In Sec.IVCthese two degrees of freedom are utilized to compute fixed points that satisfy RGT and SS constraints. In addition, the same section includes an analysis on the effect of the order of zonal harmonics on the accuracy of the results.

\section{A. Computation of Quasi-Circular Orbits}

The spacecraft crosses the equatorial plane twice during one nodal period with the vertical velocity $\dot{z}>0$ and $\dot{z}<0$, respectively. Here, we can select the surface of section $S(r, z, \dot{r}, \dot{z})=z=0, \dot{z}>0$ to find the fixed point on it. The fixed point can be then represented by a reduced state $s^{*}=\left[r^{*}, \dot{r}^{*}\right]^{T}$, because $z^{*}=0$ and the vertical velocity $\dot{z}^{*}$ can be computed from the conserved energy $E$ [Eq. [3] ], as already identified from the previous researches [20, 22, 23].

Starting from an initial guess $s_{0}=\left[r_{0}, \dot{r}_{0}\right]^{T}$ on the selected surface of section with a known (i.e., computed numerically) nodal period $T_{d}$, we first compute the map that projects any arbitrary point in the neighborhood of $\boldsymbol{s}_{0}$ to the next surface of section crossing. To achieve this it is convenient to change the independent variable of Eq. (4) from $t$ into $\tau=t / T_{d}$, where the coefficient $\tau \in[0,1]$. Accordingly, Eq. (4) can be rewritten as

$$
\left\{\begin{array}{l}
\frac{\mathrm{d} x}{\mathrm{~d} \tau}=T_{d} f\left(x, H_{z}\right), \\
\frac{\mathrm{d} l}{\mathrm{~d} \tau}=0
\end{array}\right.
$$

in which the adjoint variable $l$ satisfies the condition $l(0)=T_{d}$. In this way, the state of the dynamics $\boldsymbol{x}$ is augmented to $\boldsymbol{X}=\left[\boldsymbol{x}^{T}, \phi, l\right]^{T}$.

If both the initial guess $\boldsymbol{s}_{0}$ and its nodal period $T_{d}$ are initialized as DA variables, the integration of Eq. (11) delivers the high-order Taylor map

$$
\boldsymbol{X}_{f}=\mathcal{T}_{\boldsymbol{X}_{f}}\left(s_{0}, T_{d}\right)
$$


The computation of the fixed point requires first to determine how the nodal period changes as a function of $\boldsymbol{s}_{0}$. This is achieved by solving the PIE

$$
z_{f}=\mathcal{T}_{z_{f}}\left(s_{0}, T_{d}\right)=0
$$

(i.e., finding the zeros of the second component of map (12) ) in which $T_{d}$ is the unknown and $s_{0}$ is the vector of parameters. The application of the algorithm of Sec. IIIC, provides

$$
T_{d}=\mathcal{T}_{T_{d}}\left(s_{0}\right)
$$

i.e, the Taylor approximation of the nodal period for variations of $s_{0}$. By substituting map (14) into map (12), we obtain

$$
\boldsymbol{X}_{1}=\mathcal{T}_{\boldsymbol{X}_{1}}\left(s_{0}, \mathcal{T}_{T_{d}}\left(s_{0}\right)\right)=\hat{\mathcal{T}}_{\boldsymbol{X}_{1}}\left(s_{0}\right)
$$

The Taylor polynomial $\mathcal{T}_{\boldsymbol{X}_{1}}\left(\boldsymbol{s}_{0}\right)$ maps any point $\boldsymbol{s}_{0}$ onto the surface of section after one nodal period (explaining the use subscript "1"), approximated by $\mathcal{T}_{T_{d}}\left(s_{0}\right)$. In particular, the first and the third polynomial of $\mathcal{T}_{\boldsymbol{X}_{f}}\left(\boldsymbol{s}_{0}\right)$ are

$$
\left\{\begin{array}{l}
r_{1}=\mathcal{T}_{r_{1}}\left(s_{0}\right), \\
\dot{r}_{1}=\mathcal{T}_{\dot{r}_{1}}\left(s_{0}\right),
\end{array}\right.
$$

or in compact form

$$
s_{1}=\mathcal{T}_{s_{1}}\left(s_{0}\right)
$$

The computation of the fixed point $\boldsymbol{s}^{*}$ is achieved by solving numerically the polynomial constraint $\boldsymbol{s}_{0}-\mathcal{T}_{\boldsymbol{s}_{1}}\left(\boldsymbol{s}_{0}\right)=0$. In this work the trust-region dogleg method implemented in the MATLAB fsolve solver is used (this is algorithm we selected whenever we need to solve a set of nonlinear equations in this work). Note that only a single DA numerical integration is necessary to compute the fixed point. In addition, as the problem is reduced in polynomial form, all the derivatives required by fsolve are readily available. For these reasons we classify our method as a semi-analytical one.

A circular orbit is always found to provide a good initial guess for the computation of a fixed point (quasi-circular orbit). For illustrative purposes an initial guess corresponding to an approximate RGT-SS orbit is considered. A guess for the nodal period is obtained from $T_{0}=T_{s} n_{M} / n_{N}$, in which $n_{N}$ and $n_{M}$ are integer numbers representing the number of $n_{N}$ nodal periods in $n_{M}$ nodal days and $T_{s}$ is a sidereal day. For the following example $n_{N}=14, n_{M}=1$ are used, giving $T_{0}=7.62827715989582$. From $T_{0}$ the guess for the radial position is computed by $r_{0}=a_{0}=\left(\frac{\sqrt{\mu} T_{0}}{2 \pi}\right)^{2 / 3}=$ 1.13805800280097, which also determines the specified value of orbit energy by $E=-\mu /\left(2 a_{0}\right)=-0.439344918070439$. To compute the polar component of the angular momentum, we first determine the inclination of the circular orbit for 


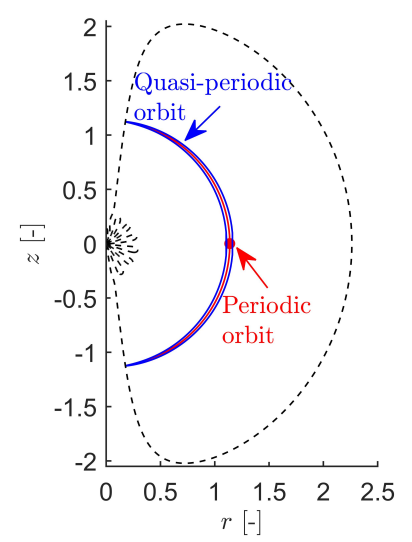

(a)

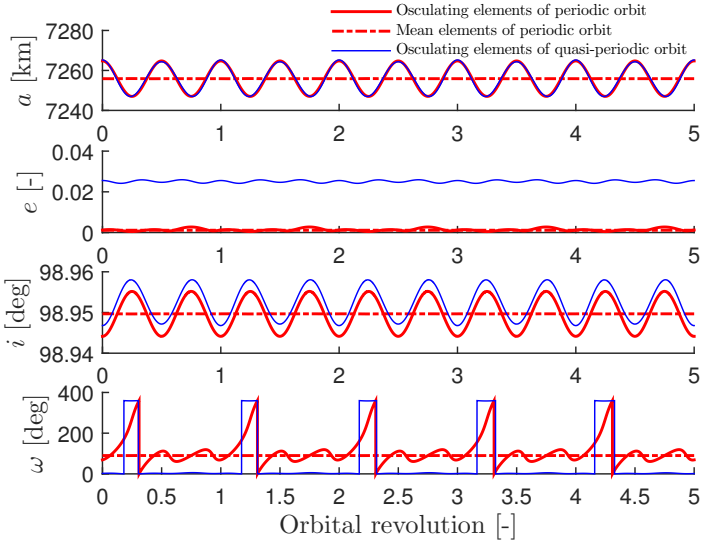

(b)

Fig. 1 Motion of fixed point (periodic orbit) and quasi-periodic orbit displayed in the configuration space (a), and their corresponding orbital elements $(a, e, i$, and $\omega)$ expressed in the ECI frame (b).

which the secular variation of RAAN induced by $J_{2}$ equates the angular velocity, $\omega_{S}$, of the Earth around the Sun: $-\frac{3}{2} J_{2} R^{2} \sqrt{\mu} a_{0}^{-7 / 2} \cos i_{0}=\omega_{S}$ [37]. Using $\omega_{S}=1.99096871 \times 10^{-7} \mathrm{~s}^{-1}$ we obtain $i_{0}=98.947912173341763 \mathrm{deg}$ and $H_{z}=\sqrt{\mu a_{0}} \cos i_{0}=-0.16592601760462$. With these guesses for $r_{0}$ and $\dot{r}_{0}$ (initialized to be 0 ) as well as the specified values of $E$ and $H_{z}$, the DA approach (at 6-th order and including $J_{15}$ zonal harmonic perturbations) computes the fixed point $\left[r^{*}, \dot{r}^{*}\right]^{T}=[1.1385091108548187,-0.0010602088953075785]^{T}$ and the nodal period $T_{d}^{*}=7.632482485088449$. The accuracy of the fixed point is $10^{-12}$, an accuracy comparable with the fully numerical methods used in [22, 23]. Correspondingly, if we display the orbit originating from the fixed point in the configuration space [i.e., $(r-z)$ rotating meridian plane of the spacecraft] and the Earth-centered inertial (ECI) frame, respectively, a very precise periodicity and frozen property can be observed, as shown in Fig. 1. In Fig.1(a), the dotted zero velocity curve places the bounds on the motion of the spacecraft for the specified energy. The forbidden realm extends outside the curve; the spacecraft moves inside the allowed region starting from the equatorial plane and oscillating up and down until the boundaries are hit. Only the fixed point-corresponding periodic orbit repeats completely (the red trajectory); whereas, starting from the same surface of section and energy level, the quasi-periodic orbits (corresponding to the central manifolds of periodic orbit plotted in blue) do not display the same periodic behavior, even though they exhibit oscillatory motion. Furthermore, if we transform the reduced states of periodic and quasi-periodic orbits into the orbital elements (semi-major axis $a$, eccentricity $e$, inclination $i$, and argument of perigee $\omega$ ) as shown in Fig.1(b) the periodicity is straightforwardly revealed from the osculating variations of the orbital elements of the periodic orbit, and its frozen property is demonstrated by a mean argument of perigee of $90 \mathrm{deg}$ and a constant mean eccentricity of order of magnitude $10^{-3}$. This explains the reason why the fixed point is referred to as the quasi-circular orbit.

It is worth mentioning that quite differently from the previous methods [22] for the search of periodic orbits under $J_{2}$ perturbation, this algorithm is the more general one; therefore, it can work for the zonal problem at any order. In 
particular, if only the even order terms $\left[J_{2 n}(n \geq 1)\right]$ are included, the fixed point has zero radial velocity (i.e., the perigee lies on the equatorial plane); however, in the presence of the odd harmonics, the radial velocity is no longer zero and the periodic orbits are actually frozen orbits with the mean argument of perigee of $90 \mathrm{deg}$.

\section{B. Families of Fixed Points}

In the previous section a fixed point was computed for the specified values of $E_{0}$ and $H_{z, 0}$. In this section DA tools are used to compute families of fixed points parameterized in terms of $E$ and $H_{z}$. This study is important to later design bounded relative motion about a chief orbit that satisfies some specific requirements.

As reported in [21], except at some critical inclinations, the periodic orbits are always stable, thus the locations of fixed points are continuous with respect to the parameters $E$ and $H_{z}$. As a result, DA techniques provide a powerful tool for the representation of families of fixed points. The algorithm starts from a known fixed point $s^{*}$ with nodal period $T_{d}^{*}$, which has been computed for specified values of $E_{0}$ and $H_{z, 0}$. All these quantities are initialized as DA variables and propagated forward to obtain

$$
\boldsymbol{X}_{f}=\mathcal{T}_{\boldsymbol{X}_{f}}\left(s^{*}, T_{d}^{*}, E, H_{z}\right)
$$

The first, second and third components of this map are

$$
\left\{\begin{array}{l}
r_{f}=\mathcal{T}_{r_{f}}\left(s^{*}, T_{d}^{*}, E, H_{z}\right), \\
z_{f}=\mathcal{T}_{z_{f}}\left(s^{*}, T_{d}^{*}, E, H_{z}\right), \\
\dot{r}_{f}=\mathcal{T}_{\dot{r}_{f}}\left(s^{*}, T_{d}^{*}, E, H_{z}\right),
\end{array}\right.
$$

on which the following constraints can be imposed

$$
\left\{\begin{array}{l}
\mathcal{T}_{r_{f}}\left(s^{*}, T_{d}^{*}, E, H_{z}\right)-r^{*}=0, \\
\mathcal{T}_{z_{f}}\left(s^{*}, T_{d}^{*}, E, H_{z}\right)=0 \\
\mathcal{T}_{\dot{r}_{f}}\left(s^{*}, T_{d}^{*}, E, H_{z}\right)-\dot{r}^{*}=0 .
\end{array}\right.
$$

Equation (20) is a set of three PIEs in which $s^{*}$ and $T_{d}^{*}$ are the unknown, and $E$ and $H_{z}$ the parameters. Its solution by means of DA techniques gives

$$
\left\{\begin{array}{l}
\boldsymbol{s}^{*}=\mathcal{T}_{\boldsymbol{s}^{*}}\left(E, H_{z}\right), \\
T_{d}^{*}=\mathcal{T}_{d}^{*}\left(E, H_{z}\right) .
\end{array}\right.
$$

The first row of map 21] is the Taylor approximation of the family of fixed points, parameterized by $E$ and $H_{z}$; 
whereas the second one delivers the corresponding nodal periods of the family. By substituting $T_{d}^{*}=\mathcal{T}_{d}^{*}\left(E, H_{z}\right)$ into map (18), the high-order approximation of the state of the family of fixed points after one nodal period is obtained as

$$
\boldsymbol{X}_{f}=\boldsymbol{X}_{1}=\mathcal{T}_{\boldsymbol{X}_{1}}\left(\mathcal{T}_{\mathbf{s}^{*}}\left(E, H_{z}\right), \mathcal{T}_{d}^{*}\left(E, H_{z}\right), E, H_{z}\right)=\hat{\mathcal{T}}_{\boldsymbol{X}_{1}}\left(E, H_{z}\right)
$$

Given any assigned values of $E$ and $H_{z}$ in the proximity of the reference values, the mere evaluation of the polynomials in Eq. 21] provides the corresponding fixed point and the associated nodal period. In Fig.2 the family of fixed points, computed with a 6-th order expansion in $45.45 \mathrm{~s}$ on a Lenovo ThinkPad with a $2.60 \mathrm{GHz}$ Intel Core i5 CPU and 4 GB RAM, is plotted for the space $E \times H_{z}=[-0.479344918070439,-0.399344918070439] \times$ $[-0.315926017604620,-0.015926017604620]$. As the orbit energy is strictly related to the semi-major axis of the orbit, for higher $E$ the fixed points have greater values of both the radial distance $r^{*}$ and nodal period $T_{d}^{*}$. On the other hand, for a fixed energy, the absolute value of polar component of the angular momentum $H_{z}$ is related to the inclination of the orbit; this explains the increase of the RAAN drift per nodal period $\Delta \Omega_{d}^{*}$ and decrease of the vertical velocity $\dot{z}^{*}$ when the absolute value of $H_{z}$ increases.

It must be noticed that Eq. 21] is a Taylor approximation of the family of fixed points, and thus it is necessary to assess its accuracy. To this aim, a fine grid is built on the $\left(E, H_{z}\right)$ space and the corresponding fixed points, computed by evaluating Eq. 21], are numerically propagated forward from $\tau=0$ to $\tau=1$. The accuracy if then assessed by analyzing

the residuals $\left|z_{1}-\mathcal{T}_{z_{1}^{*}}\left(E, H_{z}\right)\right|,\left|r_{1}-\mathcal{T}_{r_{1}^{*}}\left(E, H_{z}\right)\right|$, and $\left|\dot{r}_{1}-\mathcal{T}_{\dot{r}_{1}^{*}}\left(E, H_{z}\right)\right|$, in which $z_{1}, r_{1}$, and $\dot{r}_{1}$ are the values computed numerically after one nodal period. In Fig. 3 the logarithmic values of the residuals are plotted for the considered ranges of $H_{z}$ and $E$. It is found that around the reference value there are large regions with the error not exceeding the $10^{-8}$ order of magnitude (distance in centimeter and velocity in hundredths of a millimeter per second), and the maximum error is at the boundary of the region. Obviously, if the range of $H_{z}$ and $E$ is further increased, the error introduced by the approximation can result in an accuracy not compatible with the stringent requirements imposed by bounded relative motion design. However, when the accuracy requirement is met, the proposed approach computes an entire family of fixed points with a single DA propagation and without the need of any iterations (only polynomial inversions and composition).

\section{Constrained Fixed Points}

For practical mission design it is often important that the fixed point orbits possess some specific properties, such as RGT and/or SS. Thus, devising an algorithm capable of including additional constraints in the computation of fixed points is highly desirable. Independently from the number and type of constraints considered, the values of the fixed points are affected by the number of harmonics used to model the Earth's gravitational potential. As a result, a study on the sensitivity to the zonal harmonics order is useful to understand the value required for a sufficiently accurate design. 


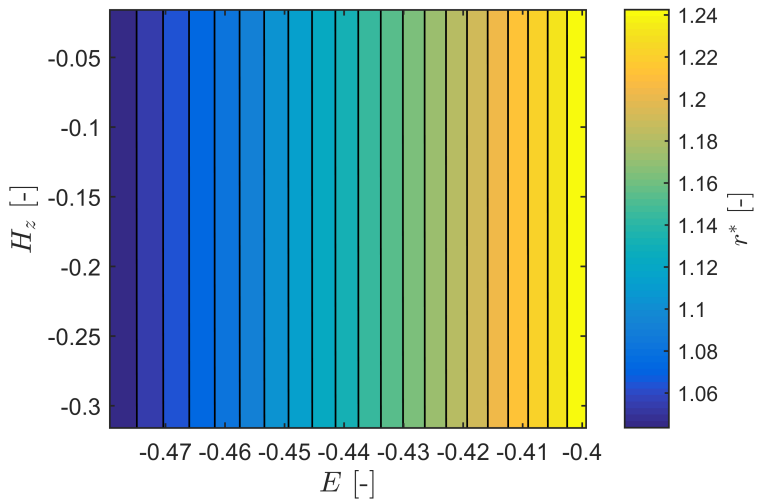

(a) $\mathcal{T}_{r^{*}}\left(E, H_{z}\right)$

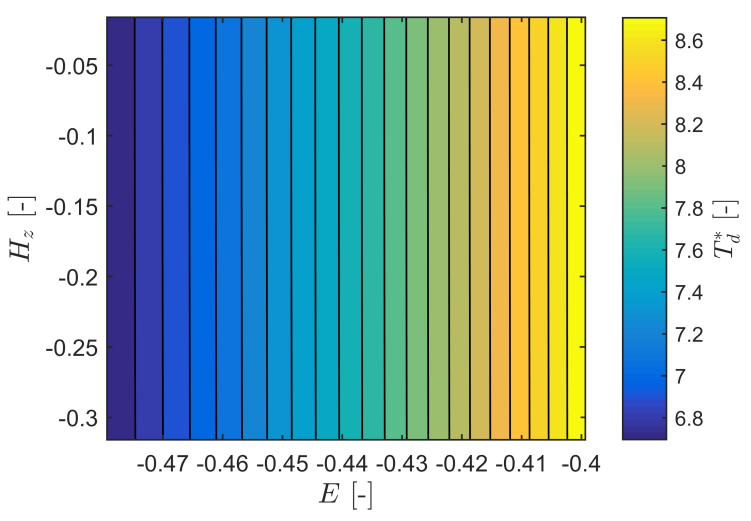

(c) $\mathcal{T}_{d}^{*}\left(E, H_{z}\right)$

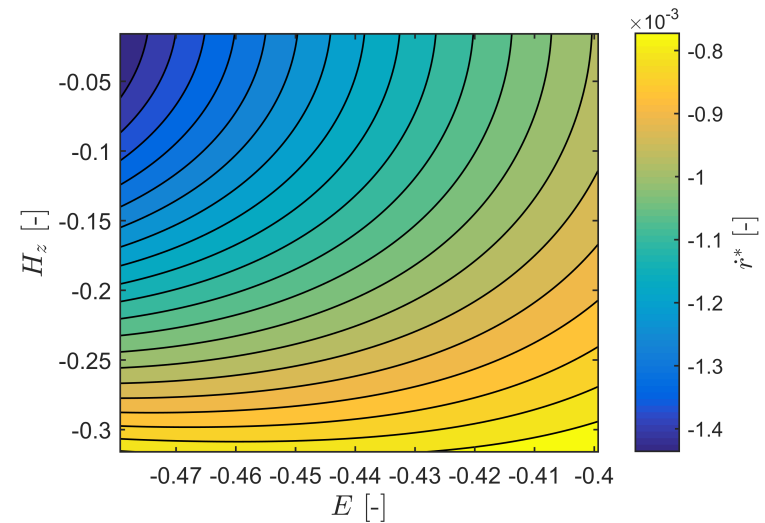

(b) $\mathcal{T}_{\dot{r}^{*}}\left(E, H_{z}\right)$

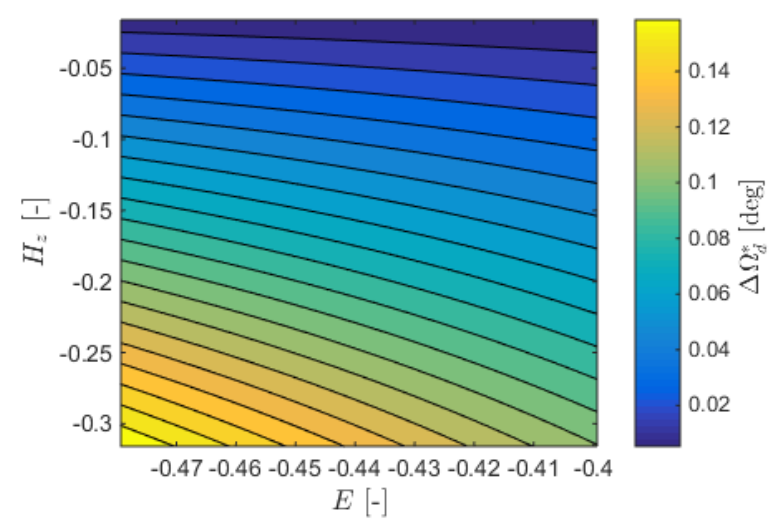

(d) $\mathcal{T}_{\Delta \Omega_{d}^{*}}\left(E, H_{z}\right)$

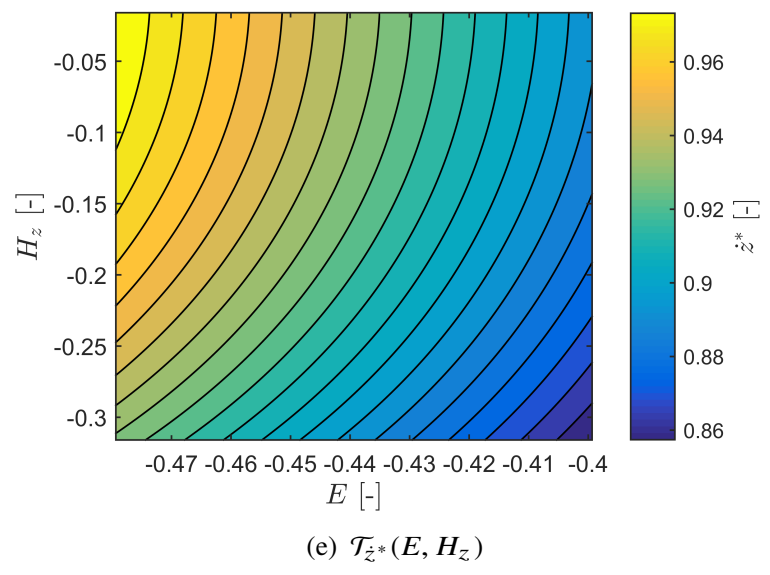

Fig. 2 Family of fixed points parametrized by $E$ and $H_{z}$, effect on radial distance $r^{*}(\mathbf{a})$; radial velocity $\dot{r}^{*}(\mathbf{b})$; nodal period $T_{d}^{*}(\mathbf{c}) ; \mathbf{R A A N}$ drift $\Delta \Omega_{d}^{*}(\mathbf{d})$; vertical velocity $\dot{z}^{*}(\mathbf{e})$. 

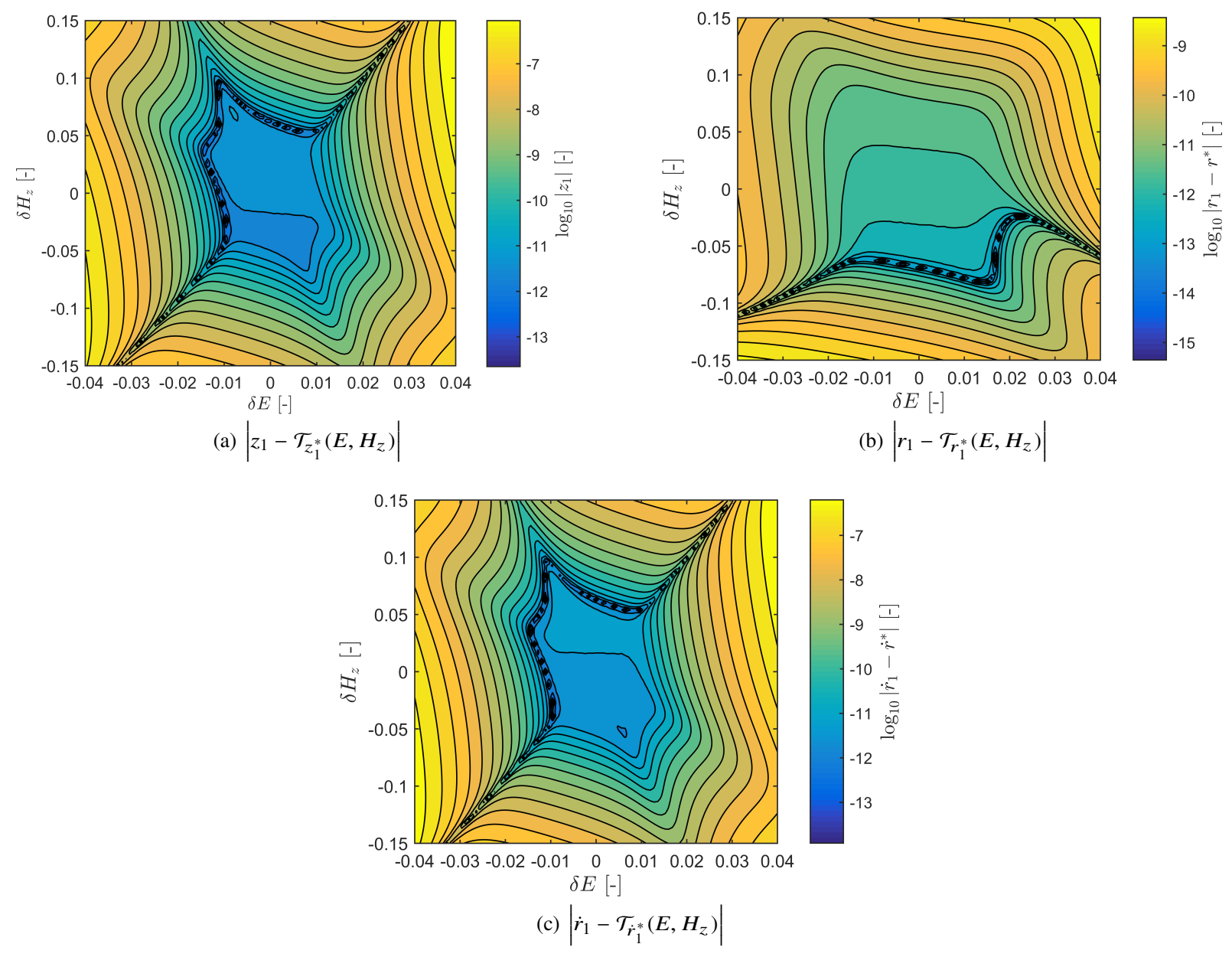

Fig. 3 Logarithmic error of the Taylor representation of the family of fixed points in the space $E \times H_{z}=$ $[-0.04,0.04] \times[-0.15,0.15]$ in terms of the achievement of the final constraint: $\left|z_{1}\right|=0(\mathbf{a}) ;\left|r_{1}-r^{*}\right|=0$ (b); $\left|\dot{r}_{1}-\dot{r}^{*}\right|=0(\mathbf{c})$. 
Both these aspects are addressed in this section, in which RGT and SS constraints are considered, as frequently used for remote sensing missions.

The RGT and SS constraints can be expressed (note that simplified ones were used in Sec.IVA) as

$$
\left\{\begin{array}{l}
n_{N}\left(\omega_{E} T_{d}-\Delta \Omega_{d}\right)-2 \pi n_{M}=0 \\
\Delta \Omega_{d} / T_{d}-\omega_{S}=0
\end{array}\right.
$$

in which $\omega_{E}=7.2921159 \times 10^{-5} \mathrm{~s}^{-1}$ is the rotation rate of the Earth. In Sec. IVB the family of fixed points has been parametrized by $E$ and $H_{z}$; the application of both constraints 23 will remove these two degrees of freedom and provide with a single fixed point. The RGT constraint is addressed first. The Taylor approximations for the nodal period $T_{d}^{*}=\mathcal{T}_{d}^{*}\left(E, H_{z}\right)$ and RAAN drift $\Delta \Omega_{d}^{*}=\mathcal{T}_{\Delta \Omega_{d}^{*}}\left(E, H_{z}\right)$, obtained by evaluating in DA Eq. (7), is substituted in the expression of the RGT constraint, resulting in

$$
n_{N}\left[\omega_{E} \mathcal{T}_{d}^{*}\left(E, H_{z}\right)-\mathcal{T}_{\Delta \Omega_{d}^{*}}\left(E, H_{z}\right)\right]-2 \pi n_{M}=0
$$

Equation 24) is a PIE in which $E$ is the unknown and $H_{z}$ is the parameter. The solution of Eq. 24 provides the Taylor approximation $E=\mathcal{T}_{E}\left(H_{z}\right)$, which is substituted back in maps (21) and 22), delivers the 1D family of quasi-circular RGT orbits satisfying the $n_{M}-n_{N}$ repetition pattern. The subsequent imposition of the SS constraint removes the remaining degree of freedom, $H_{z}$, and a single quasi-circular RGT-SS orbit is obtained. Both the family of quasi-circular RGT orbits and the quasi-circular RGT-SS orbit are plotted in Fig.4, where the $J_{15}$ perturbations are considered. The family of RGT orbits are displayed by the solid line, on which the point possessing both the RGT and SS proprieties can be located.

It is worth noting that the RGT and SS constraints are used in the section only for illustrative purposes and that they can straightforwardly substituted by different ones, such as specific values of the semi-major axis and/or inclination. However, as reported by Broucke [21], the periodic orbit should be far from the critical inclination one in case of the appearance of the bifurcation which is beyond the scope of our current work.

To assess how the number of harmonics affects the orbit design, the following numerical simulations are presented. Figure 5 displays the variation of the RGT family of fixed points when different zonal terms are taken into account. In Fig. 6 the same analysis is offered by plotting the corresponding orbital elements. It is clear that stable results are achieved in terms of all the relevant quantities for $n \geq 10$. When the SS constraint is added both the $E$ and $H_{z}$ degrees of freedom are removed and therefore there is only one possible pair of $\left(E, H_{z}\right)$. In Fig. 7 the variation of relevant quantities as function of the selected order for the zonal harmonics is plotted, showing also in this case that convergence is reached for $n \geq 10$. 


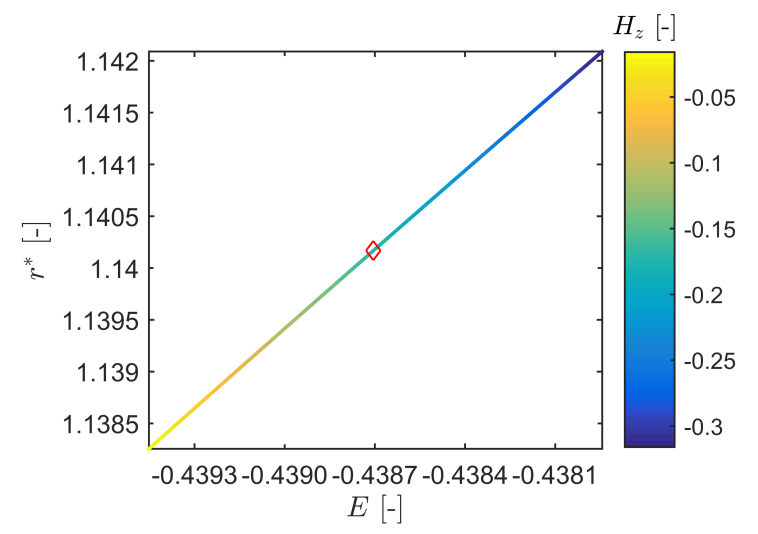

(a)

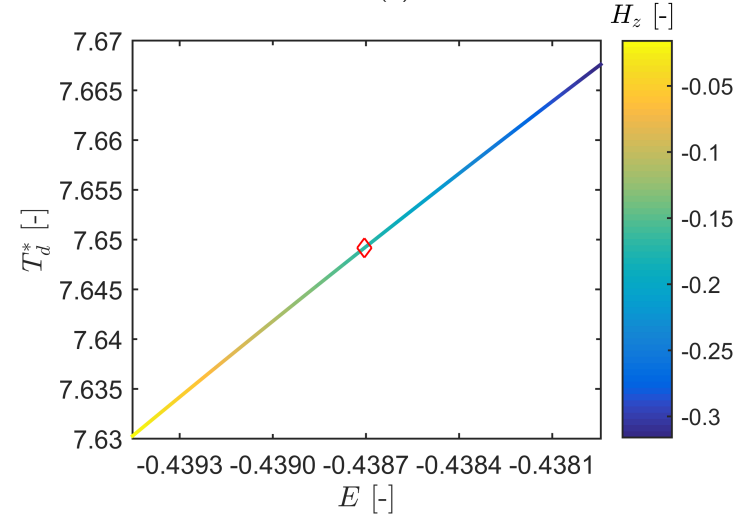

(c)

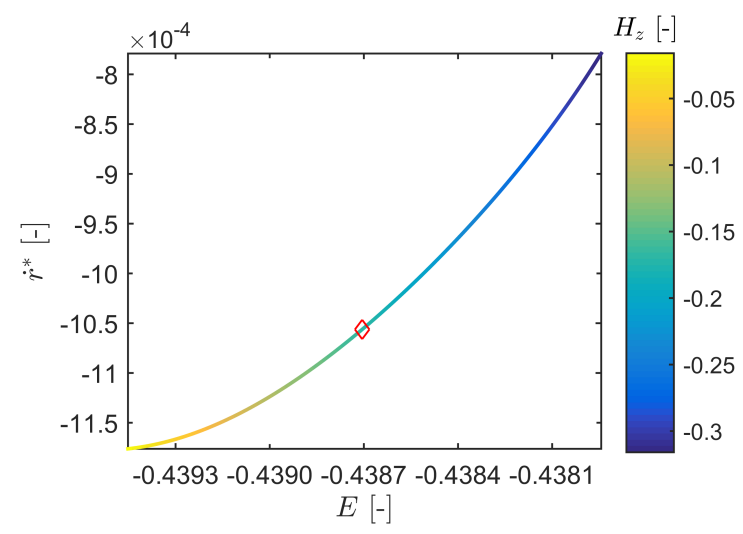

(b)

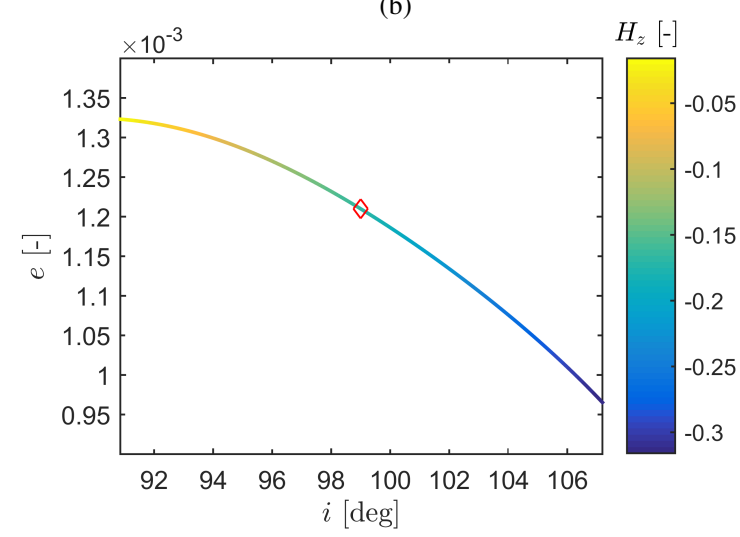

(d)

Fig. 4 Family quasi-circular RGT orbits (solid line) and quasi-circular RGT-SS orbit (diamond). 

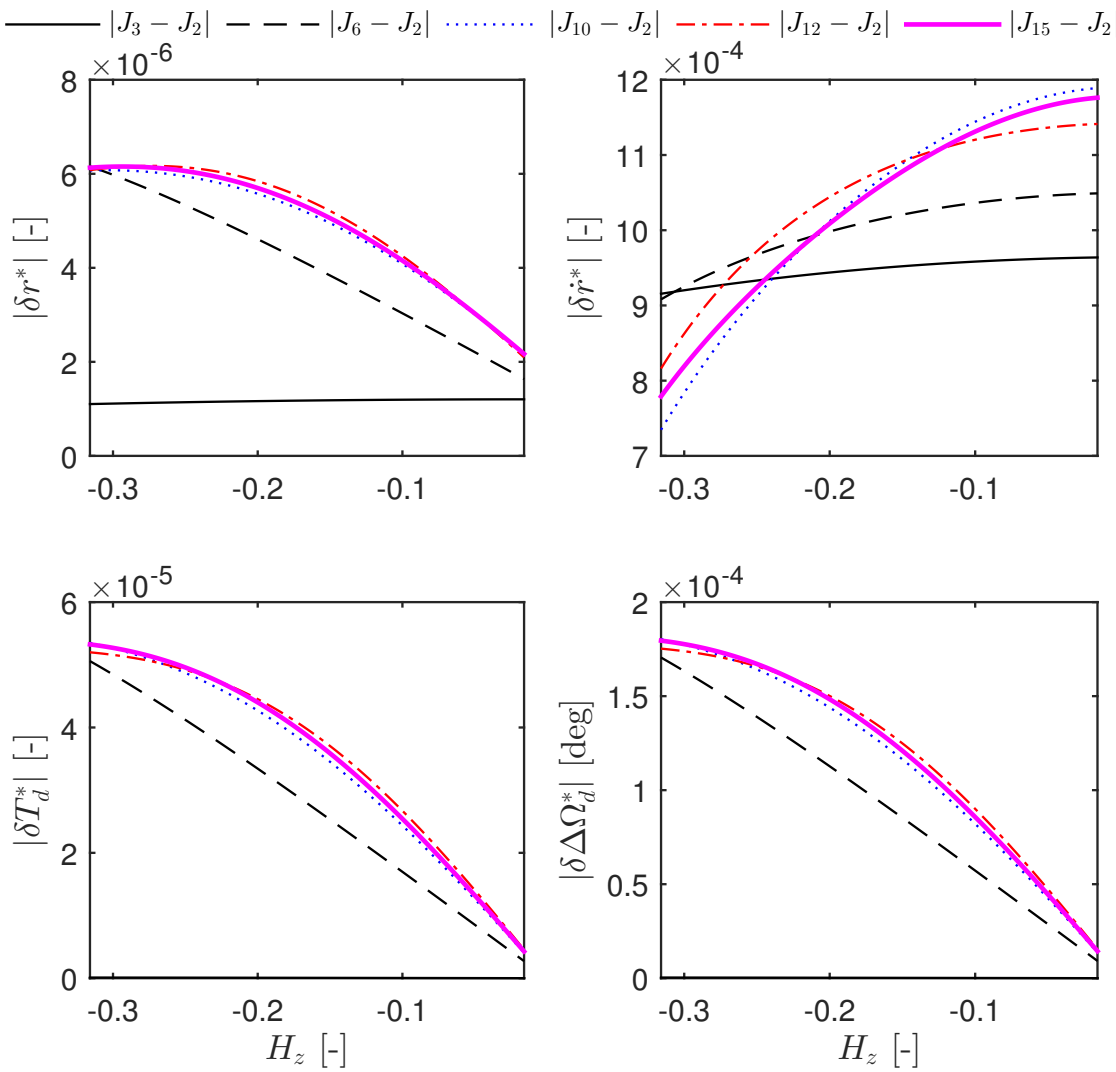

Fig. 5 Deviation of the fixed point quantities from $J_{2}$ perturbation under higher-order $\left[J_{n}(n=3, \ldots, 15)\right]$ perturbations.

From Figs. 5, 6 and 7it can be concluded that all the fixed point quantities show a stable behavior for values of $n \geq 10$, as already shown in [33]. This reveals the significance of the higher-order perturbations in dealing with the zonal problem and it is actually the reason why the $J_{15}$ perturbations are always adopted in this work.

\section{Design of Long-Term Bounded Relative Orbits}

In this section, the constraints for designing bounded relative motion are first introduced (Sec. VA). These are followed by the presentation of the algorithm to calculate the high-order approximation of Poincaré maps and how these are used to efficiently propagate perturbed initial conditions for thousands of revolutions (Sec. VB B). Afterwards, the algorithm to efficiently compute invariant curves around the periodic orbit is detailed together with the presentation of some bounded relative motions in Sec. VII Section V|R introduces a method to numerically refine the results for cases in which the high-order Poincaré maps are not sufficiently accurate and Sec.V|[B introduces an optimization method for the design of bounded relative orbits with some specific requirements. 

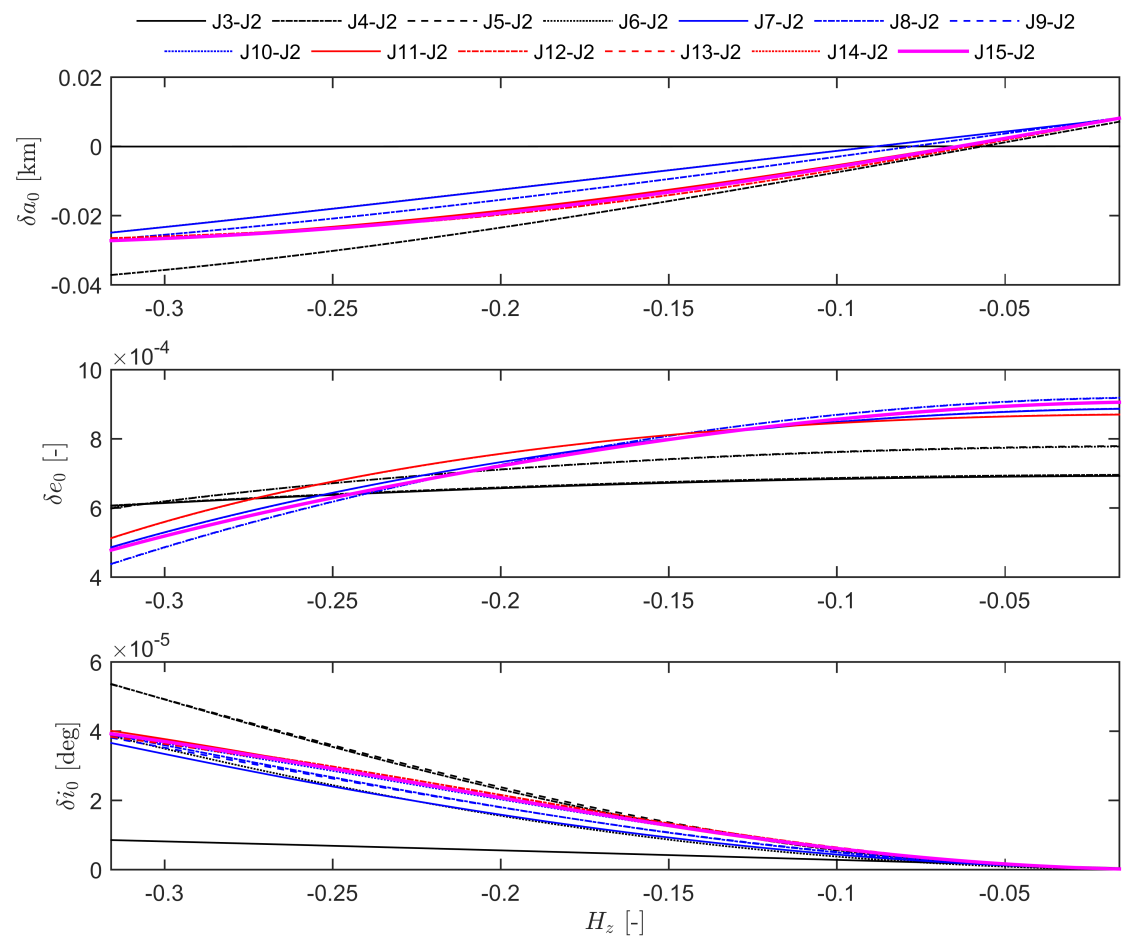

Fig. 6 Deviation of orbital elements corresponding to the fixed point from $J_{2}$ perturbation under higher-order $\left[J_{n}(n=3, \ldots, 15)\right]$ perturbations.
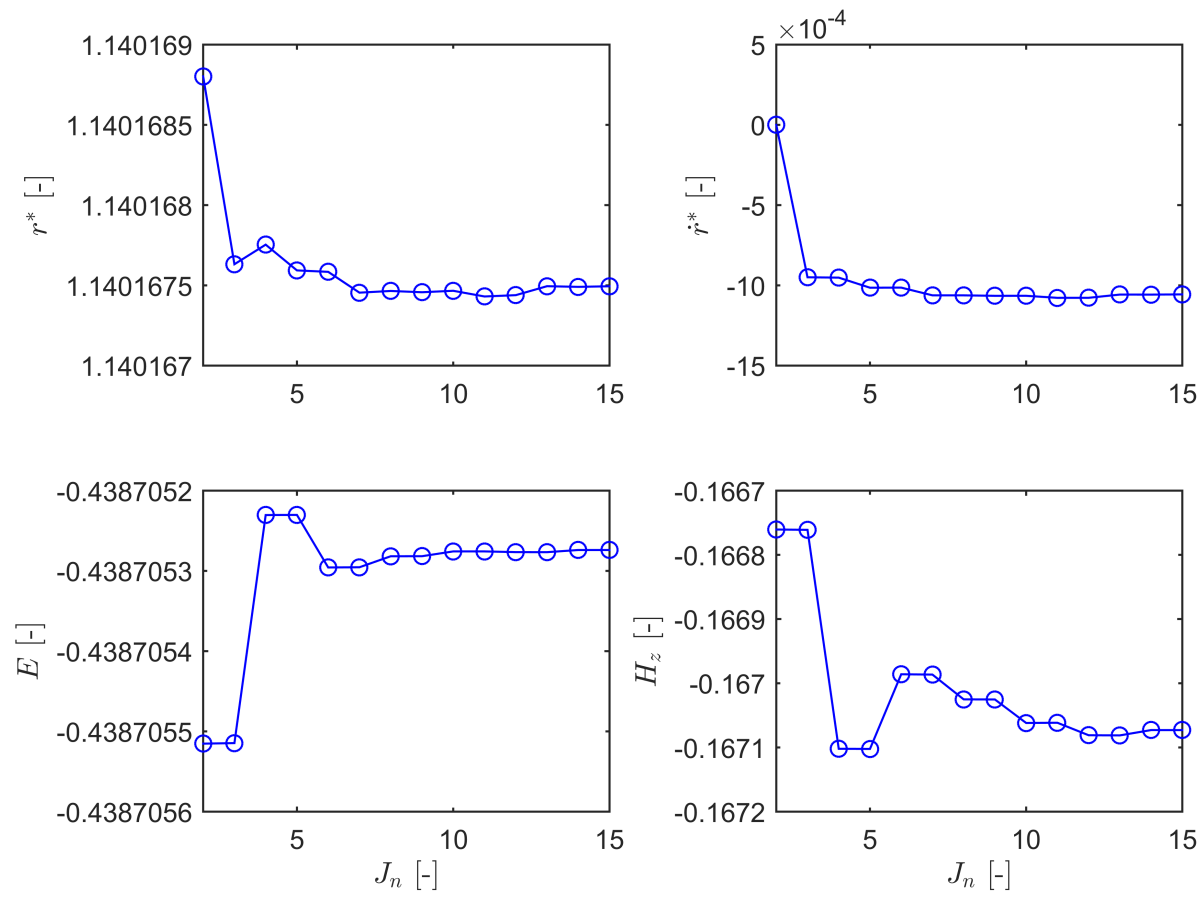

Fig. 7 Variations of fixed point quantities with the order of $J_{n}(n=2, \ldots, 15)$ zonal harmonic perturbations. 


\section{A. Constraints for Bounded Relative Orbits}

Bounded relative motion in the zonal problem is generated by the relative dynamics of two spacecraft that share both the same average nodal period and RAAN drift over one nodal period [22]. As already numerically demonstrated by Xu et al. [22], two fixed points cannot generate bounded relative motion; this is only possible between the combination of one periodic and one quasi-periodic orbit, as well as the combination of two quasi-periodic orbits. To achieve long-lasting bounded relative motion, it is first required to consider the long-periodic effect of the perturbations. That is to say, for any initial conditions in the zonal problem, it is observed that the resulting $T_{d}$ and $\Delta \Omega_{d}$ exhibit periodic-oscillation behaviors. Accordingly, the averaged values of $T_{d}$ and $\Delta \Omega_{d}$ are

$$
\bar{T}_{d}=\lim _{N \rightarrow \infty} \frac{1}{N} \sum_{i=1}^{N} T_{d}^{i} \text { and } \Delta \bar{\Omega}_{d}=\lim _{N \rightarrow \infty} \frac{1}{N} \sum_{i=1}^{N} \Delta \Omega_{d}^{i}
$$

in which $T_{d}^{i}$ and $\Delta \Omega_{d}^{i}$ are, respectively, the values of $T_{d}$ and $\Delta \Omega_{d}$ in the $i$-th revolution. In particular, $T_{d}$ and $\Delta \Omega_{d}$ of the fixed point are constant and hereinafter referred to as $T_{d}^{*}$ and $\Delta \Omega_{d}^{*}$. To generate the bounded relative orbits, the following two constraints on nodal period and drift in RAAN per nodal period are imposed

$$
\bar{T}_{d}=T_{d}^{*} \text { and } \Delta \bar{\Omega}_{d}=\Delta \Omega_{d}^{*}
$$

Using the DA techniques, we could easily impose these constraints by the repetitive evaluation of high-order Poincaré maps, as explained in Sec.VIC

\section{B. High-Order Poincaré Maps}

To compute the central manifolds (i.e., quasi-periodic orbits) around the fixed point, a DA-based method is proposed to generate a high-order Poincaré map that maps any point on the surface of section to the same surface of section after one revolution. The algorithm starts from the availability of a fixed point, e.g. the one satisfying the RGT and SS constraints of Sec.IVIC The full state vector $\boldsymbol{X}=\left[r, z, \dot{r}, \dot{z}, \phi, T_{d}\right]^{T}$ and $\dot{\phi}$ are all initialized as DA variables and the dynamics (11) are propagated from $\tau=0$ to $\tau=1$. This delivers the Taylor approximation

$$
\boldsymbol{X}_{f}=\mathcal{T}_{\boldsymbol{X}_{f}}\left(r, z, \dot{r}, \dot{z}, \phi, \dot{\phi}, T_{d}\right)
$$

Note that, differently from before, here $E$ and $H_{z}$ are dealt as dependent quantities that can be calculated from the state vector and $\dot{\phi}$. The first step is to remove the $T_{d}$ degree of freedom by solving for

$$
\mathcal{T}_{z_{f}}\left(r, z, \dot{r}, \dot{z}, \phi, \dot{\phi}, T_{d}\right)=0
$$


thus delivering

$$
T_{d}=\mathcal{T}_{T_{d}}(r, z, \dot{r}, \dot{z}, \phi, \dot{\phi})
$$

which is then substituted back into map 27] and provides

$$
\boldsymbol{X}_{f}=\boldsymbol{X}_{1}=\mathcal{T}_{\boldsymbol{X}_{1}}(r, z, \dot{r}, \dot{z}, \phi, \dot{\phi})
$$

The map (30) projects any point in the neighborhood of the fixed point $\boldsymbol{X}^{*}$ (note that $\phi^{*}$ can be arbitrarily selected and that its variation, not its value, is fixed in a nodal period) on the surface of section $z=0$ after one revolution, and the map (29) provides the required time. For this reason we also refer to $\boldsymbol{X}_{1}=\mathcal{T}_{\boldsymbol{X}_{1}}(r, z, \dot{r}, \dot{z}, \phi, \dot{\phi})$ as the 1-turn map. If the initial point is initially placed on the surface of section, then map 30 is nothing else than the high-order expansion of the Poincaré map, and map (29) provides the nodal period. The computation of this map requires performing a DA propagation in 7 variables and thus it is much more computationally expensive than a floating point propagation (e.g., at order 6 it takes as much as 94.05 s). However, due to the nature of perturbed Keplerian motion, the 1-turn map is close to an identity and this property enables its repetitive use to project a point on the surface of section for many (thousands of) revolutions [26]. Thus, while the computation of the 1-turn map is computationally intensive, the propagation of a point for multiple revolutions is extremely efficient as it just involves the evaluation of polynomials.

Figure 8 illustrates how an arbitrary initial deviation from the fixed point $\delta \boldsymbol{X}_{0}$ can be repeatedly mapped on the surface of section, thus obtaining the sequence $\delta \boldsymbol{X}_{0}, \boldsymbol{\delta} \boldsymbol{X}_{1}, \ldots, \delta \boldsymbol{X}_{N}$. It is worth highlighting that any perturbed initial condition has a different nodal period, and thus the intersection with the surface of section occurs at different times.

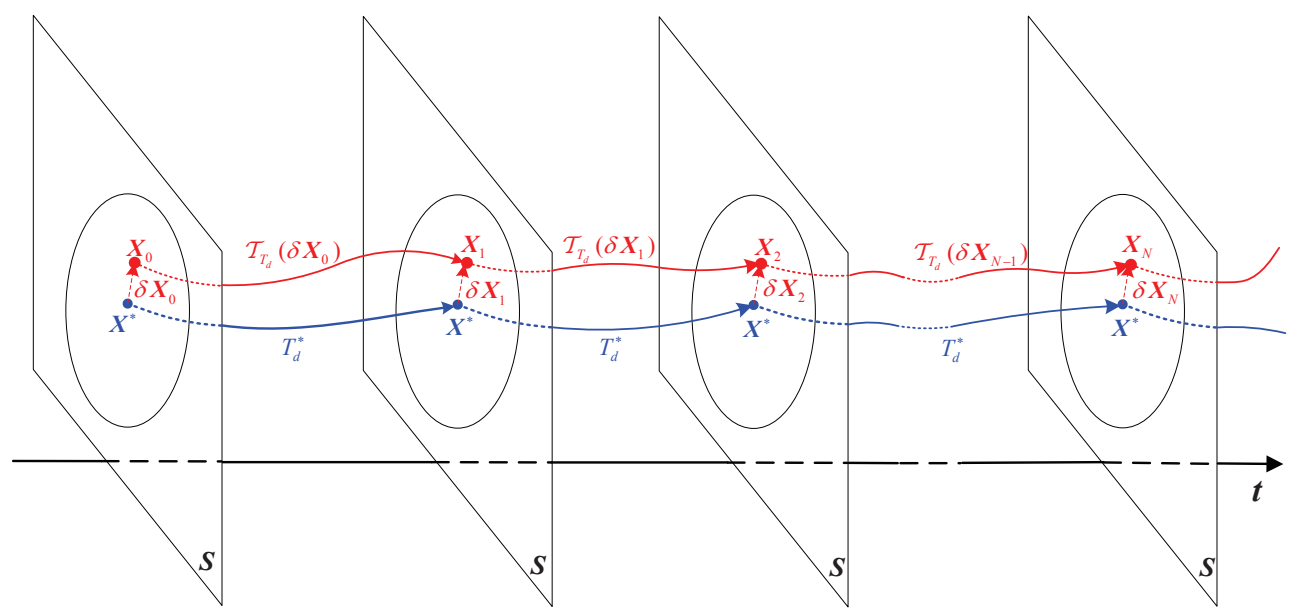

Fig. 8 Schematic illustration of the evaluation of the high-order Poincaré map to approximate the solution at each surface of section crossing.

The sequence $\boldsymbol{X}_{0}, \boldsymbol{X}_{1}, \ldots, \boldsymbol{X}_{N}$ is accurately computed by the high-order Poincaré map as long as its truncation error is small when evaluated in the deviations $\delta \boldsymbol{X}_{0}, \boldsymbol{\delta} \boldsymbol{X}_{1}, \ldots, \boldsymbol{\delta} \boldsymbol{X}_{N}$, in which $\boldsymbol{\delta} \boldsymbol{X}_{i}=\boldsymbol{X}_{i}-\boldsymbol{X}^{*}$. This map is generally valid in 
the zonal problem when orbits with limited eccentricity are considered, such that the precession of the argument of perigee causes limited deviations from the expansion point (i.e., the fixed point). In Wittig et al. [38] and Armellin and Lizia [32] it was illustrated that it was possible to estimate the size (the order of magnitude) of the region in which the truncation error of the Taylor approximation was lower than a prescribed value relying exclusively on the use of the coefficients of the map. However, in this work we evaluate the accuracy of the Taylor representation by numerically validating the results, as this method provides a more precise assessment and it represents a viable approach during the mission design phases. As will be shown in the following sections, it is found that a 6-th order expansion allows us to accurately map meaningful perturbed initial conditions for thousands of revolutions in the $J_{15}$ zonal problem.

As a final note it is worth mentioning 1-turn map method is particularly suited for autonomous dynamics, like the zonal problem. The extension to non-autonomous systems, e.g. to include the effect of luni-solar perturbations or full geopotential, is complicated by the need of computing accurate Taylor approximations of trigonometric functions for entire revolutions of their arguments, e.g. an entire revolution of the Greenwich sidereal time [39].

\section{Generation of Bounded Relative Motion}

\section{Quasi-Periodic Invariant Curves}

The map $\boldsymbol{X}_{1}=\mathcal{T}_{\boldsymbol{X}_{1}}(r, z, \dot{r}, \dot{z}, \phi, \dot{\phi})$ has 6 degrees of freedom, while only two constraints are needed to design the bounded relative motion, i.e. Eq. 26. The additional degrees of freedom can be used to parameterize the family of quasi-periodic invariant curves, i.e. the curves that satisfy the bounded relative motion constraints. In particular, $\delta r$ is used to parametrize the invariant curves, $\delta \phi$ is selected to shape the cross-track component of the relative motion between the chief and the deputy $(\delta \phi=0.5 \mathrm{deg}$ is used in this section and more details are provided in Sec. VDP , it is assumed that $\delta z=\delta \dot{r}=0$, and $\delta \dot{z}$ and $\delta \dot{\phi}$ are solved to match the constraints provided by Eq. 26.).

The procedure to find $\delta \dot{z}$ and $\delta \dot{\phi}$ is rather simple. The high-order Poincaré map is evaluated for a large number of times (to allow for multiple revolutions of $T_{d}$ and $\Omega_{d}$ ) starting from assigned values of $\delta r$ and $\delta \phi$ and initial guesses $\delta \dot{z}=\delta \dot{\phi}=0$. This delivers the sequence $T_{d}^{i}, \Delta \Omega_{d}^{i}$, with $i=1, \ldots, N$. These time series are analyzed to identify the approximate integer number of revolutions per period for $T_{d}$ and $\Delta \Omega_{d}, N_{T_{d}}$ and $N_{\Delta \Omega_{d}}$, and Eqs. (25) are approximated by

$$
\bar{T}_{d}=\frac{1}{N_{T_{d}}} \sum_{i=1}^{N_{T_{d}}} T_{d}^{i} \text { and } \Delta \bar{\Omega}_{d}=\frac{1}{N_{\Delta \Omega_{d}}} \sum_{i=1}^{N_{\Delta \Omega_{d}}} \Delta \Omega_{d}^{i}
$$

Note that multiple periods can be identified within the generated time series, and the values of $\bar{T}_{d}$ and $\Delta \bar{\Omega}_{d}$ are actually obtained by averaging the slightly different results obtained for the different periods. Once $\bar{T}_{d}$ and $\Delta \bar{\Omega}_{d}$ are computed, Eqs. (26) are solved using the MATLAB fsolve function. As this algorithm requires only polynomial evaluations we classify our approach as semi-analytical. The main advantages of the approach are its simplicity and efficiency. The computational time for the determination of invariant curves is dominated by the time required for building the 6-th 
Table 1 Initial conditions for the chief and deputy on the invariant curves to generate the bounded relative motion.

\begin{tabular}{ccccc}
\hline \multirow{2}{*}{ Orbital states } & \multicolumn{2}{c}{ Case A: periodic orbit vs. quasi-periodic orbit } & \multicolumn{2}{c}{ Case B: quasi-periodic orbit vs. quasi-periodic orbit } \\
& Chief $(\delta r=0)$ & Deputy $(\delta r=0.09)$ & Chief $(\delta r=0.01)$ & Deputy $(\delta r=0.07)$ \\
\hline$r,[-]$ & 1.140167494395865 & 1.230167494395865 & 1.150167494395865 & 1.210167494395865 \\
$z,[-]$ & 0 & 0 & 0 & 0 \\
$\dot{r},[-]$ & -0.00105621369827 & -0.00105621369827 & -0.00105621369827 & -0.00105621369827 \\
$\dot{z},[-]$ & 0.925189539301464 & 0.855032970538899 & 0.917107123913856 & 0.870147342945336 \\
$\phi,[-]$ & 0 & 0.008726646259972 & 0.008726646259972 & 0.008726646259972 \\
\hline
\end{tabular}

order expansion of the Poincaré map (only one map required for the entire family), as its multiple evaluations are very efficient; e.g., requiring only about a second when $N=6,000$. Figure 9 shows the family of invariant quasi-periodic curves obtained by running the semi-analytical procedure for increasing values of $\delta r$. Each curve is characterized by a specific value of $H_{z}$ or $E$, which is readily available once $\delta \dot{z}$ and $\delta \dot{\phi}$ are solved for. The $H_{z}$ values are highlighted directly in Fig.9, whereas the relation between $H_{z}$ and $E$ is shown in Fig. 10 . Note that fsolve takes in general 3-4 iterations to compute an invariant curve when the solution obtained for a specified value of $\delta r$ is used as the first guess for the next value on the grid to facilitate convergence.

Points belonging to different curves in Fig. 9 are characterized by different values of $E$ and $H_{z}$, but they all satisfy $\bar{T}_{d}=T_{d}^{*}$ and $\Delta \bar{\Omega}_{d}=\Delta \Omega_{d}^{*}$, as shown for the $\delta r=0.09$ case in Fig. 11 . Thus, any point belonging to an invariant curve satisfies the bounded relative motion conditions not only with respect to the periodic orbit, but also with respect to any other point belonging to an invariant curve. As a result, Fig. 9 provides us with all of the possible combinations that yield bounded relative orbits (as long as the DA-based high-order maps are accurate). Specifically, the initial values of the chief and deputy can be assigned using the combination of a periodic orbit and a quasi-periodic orbit or the combination of any two quasi-periodic orbits (as well as the trivial combination of different points belonging to the same invariant curve).

Figure 12 shows, for 6,000 revolutions (more than 400 days), the relative motion between a chief initialized on a periodic curve and a deputy initialized on a quasi-periodic orbit with $\delta r=0.09$. Their initial conditions, expressed as $[r, z, \dot{r}, \dot{z}, \phi]^{T}$ in normalized units, are given in the Case A by Table 1 . Figure 13 illustrates the evolution of the relative distance between the two spacecraft in the same time frame. It is worth noting that while the initial conditions are calculated with the semi-analytical procedure, the plots are obtained by numerically propagating the initial conditions, thus proving the suitable accuracy of a 6-th order DA map. In particular, the absence of drift in the radial-along-track plane (refer to Fig. 12(b)], is a further proof of the high accuracy achieved in the computation of the initial values. Remarkably, even when the propagation time is further extended, e.g. to 10 years, the initial values still provide an accurate bounded relative motion, as displayed in Fig. 14

When both the chief and deputy are initialized on quasi-periodic invariant curves, results as those presented in 


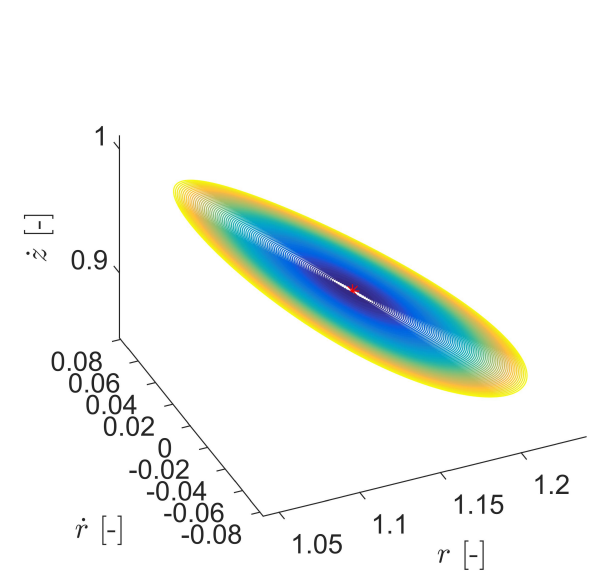

(a)

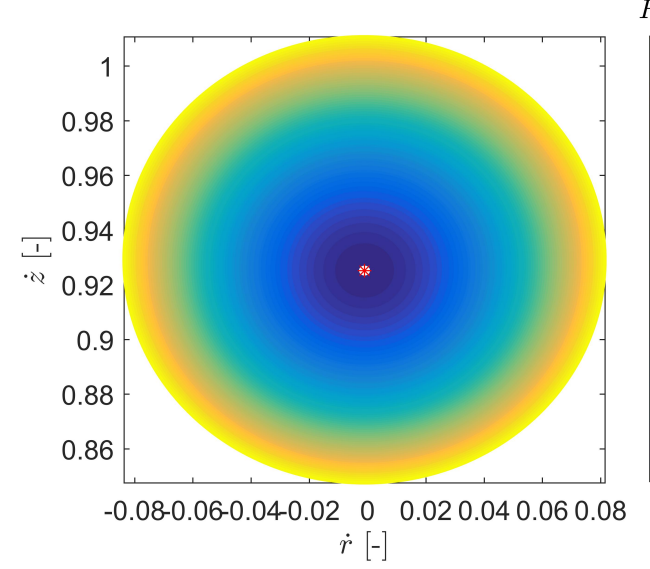

(c)
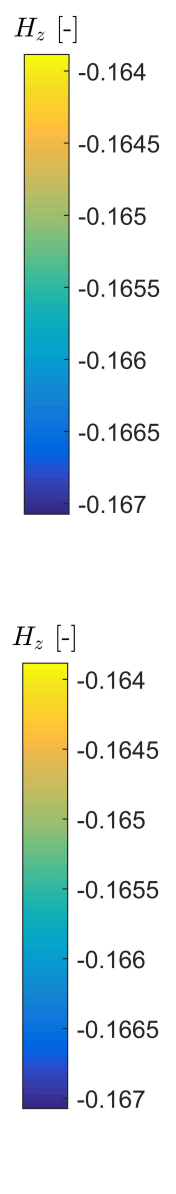

Fig. 9 Invariant curves of the Poincaré maps corresponding to different values of $H_{z}$ generated with the DA-based method.

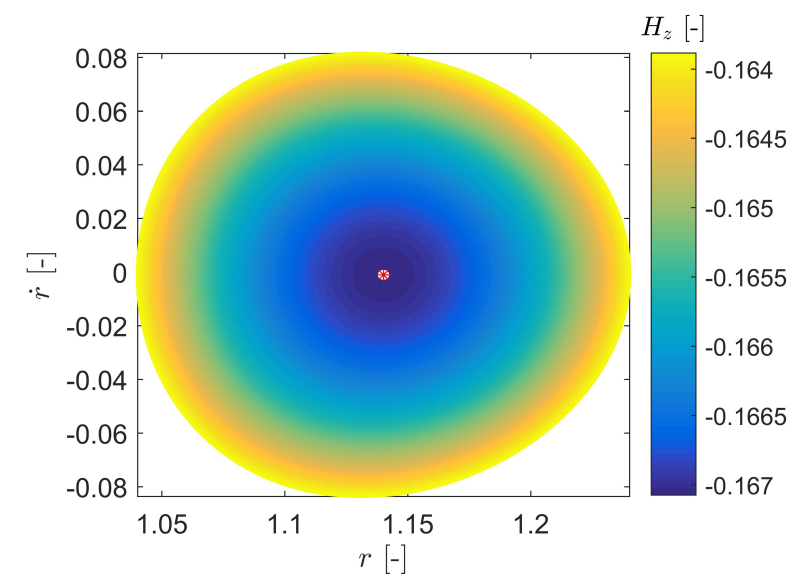

(b)

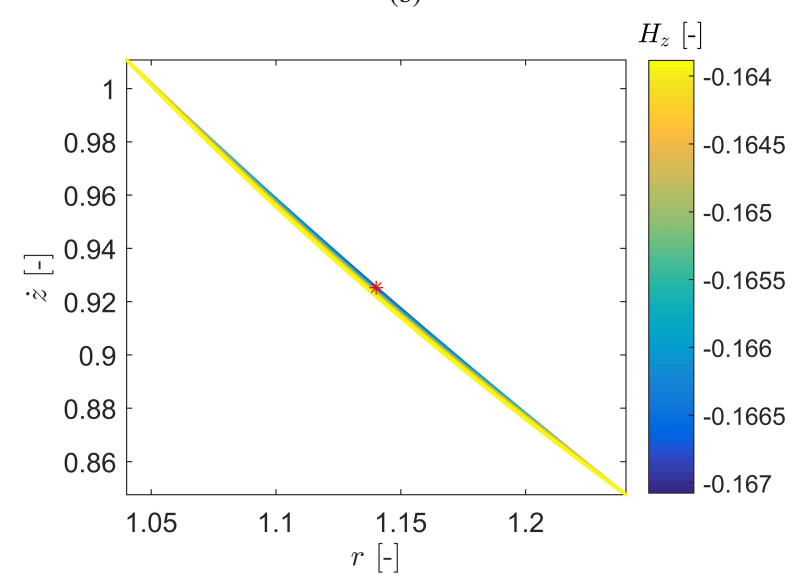

(d) 


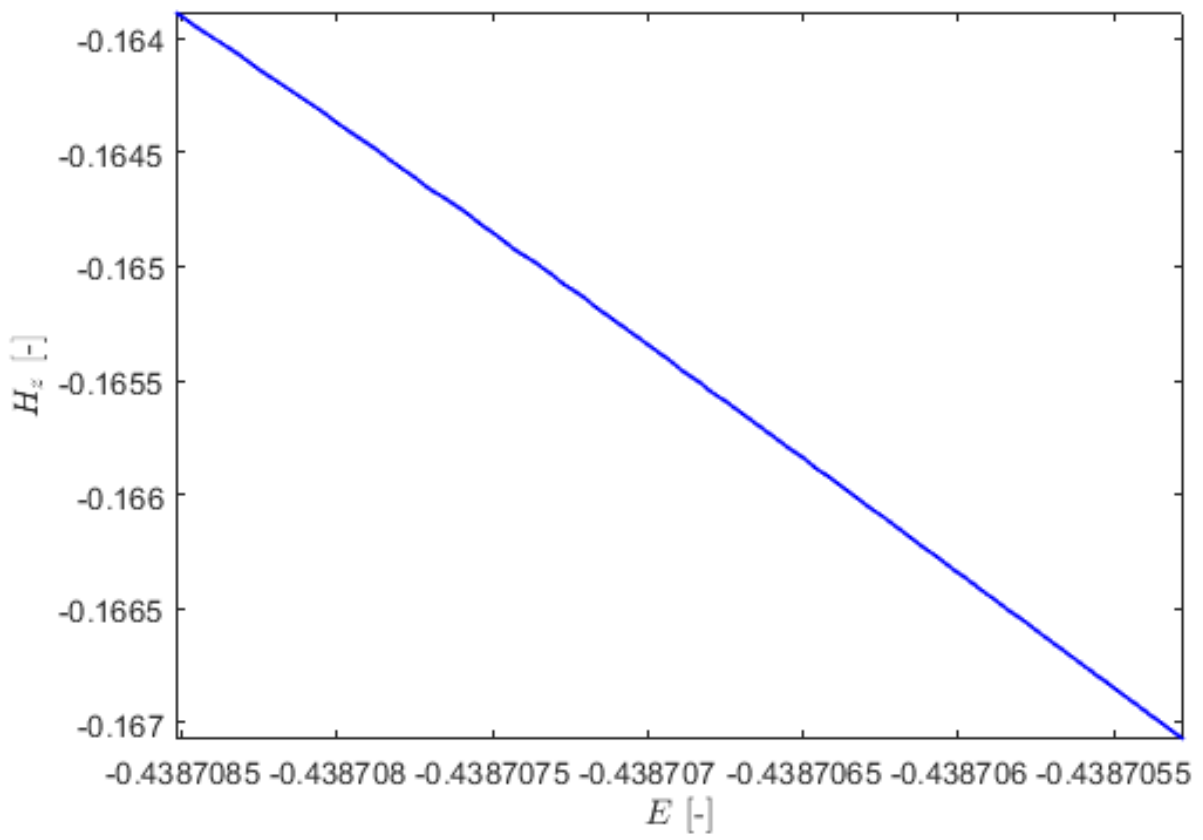

Fig. 10 One-to-one correspondence of $H_{z}$ and $E$ for invariant curves generated with the DA-based method.

Figs. 15 and 16 are obtained. In this example $\delta r=0.01$ and $\delta r=0.07$ were used for the chief and deputy, respectively, resulting in the initial conditions, reported as Case B in Table 1. Figure 15 depicts the long-term evolution of $T_{d}$ and $\Delta \Omega_{d}$, showing that the chief and deputy share the same average values of these quantities. Consequently, a long-term bounded relative motion is achieved, as highlighted in Fig. 16

To assess how the tesseral and sectorial perturbations affect the solutions obtained in the zonal problem, the solutions from Table 1 are validated in the $J_{4,4}$ model. Figure 17 shows the variation of relative distance starting from the initial conditions of Case A and Case B with respect to different initial longitudes $\lambda_{0}$. It can be observed that a drift in the relative distances shows up and that its magnitude is dependent on the initial longitude. Although higher-degree zonal terms have been used in the design of bounded relative orbits, other neglected perturbations (such as the tesseral and sectorial harmonic terms) could significantly contribute to alter the relative motion, and thus suitable formation maintenance strategies will be required.

\section{Numerical Refinement of Large Amplitude Relative Motion}

The main limitation of the proposed DA-based algorithm is the accuracy of the Taylor approximation of the Poincaré map. This limitation shows up when searching for relative motion with large amplitudes, e.g. when $\delta r=0.11$ is used (note that $\delta r \approx 1$ is the estimated order of magnitude at which the truncation error of the 6-th order expansion of the Poincaré map is $\approx 10^{-8}$, according to the coefficient-based method presented in Armellin and Lizia [32]). This is clearly shown in the top panels of Fig. 18 in which a drift in relative distance starts to develop after roughly 50 days. One way 


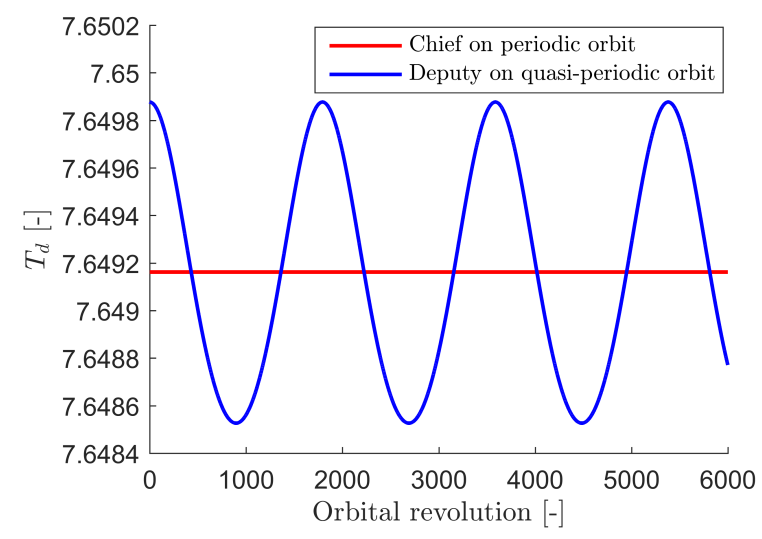

(a)

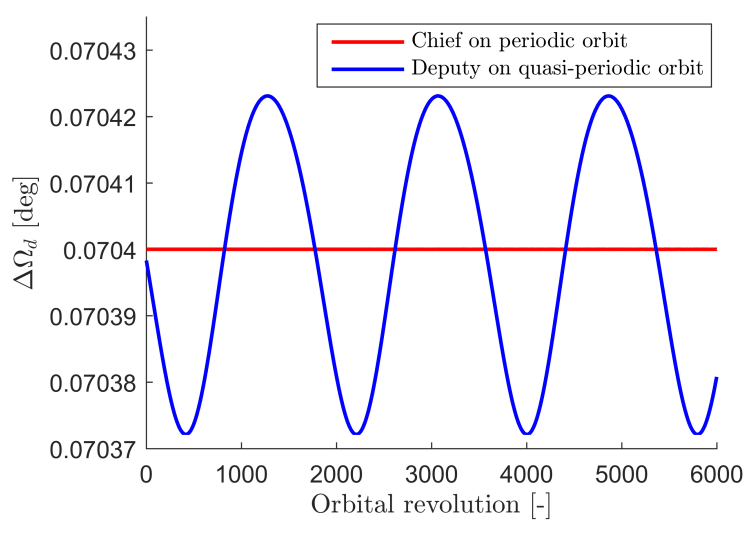

(b)

Fig. 11 Variation of $T_{d}$ (left) and $\Delta \Omega_{d}$ (right) of orbits on the invariant curves with the periodic orbit and quasi-periodic orbit (identified by $\delta r=0.09$ ).

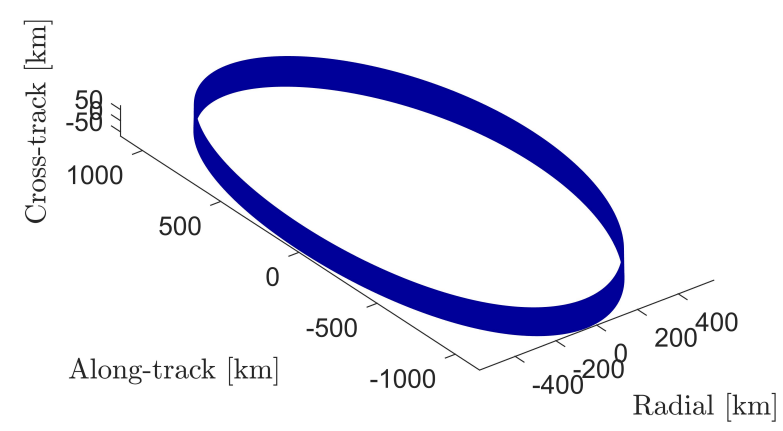

(a)

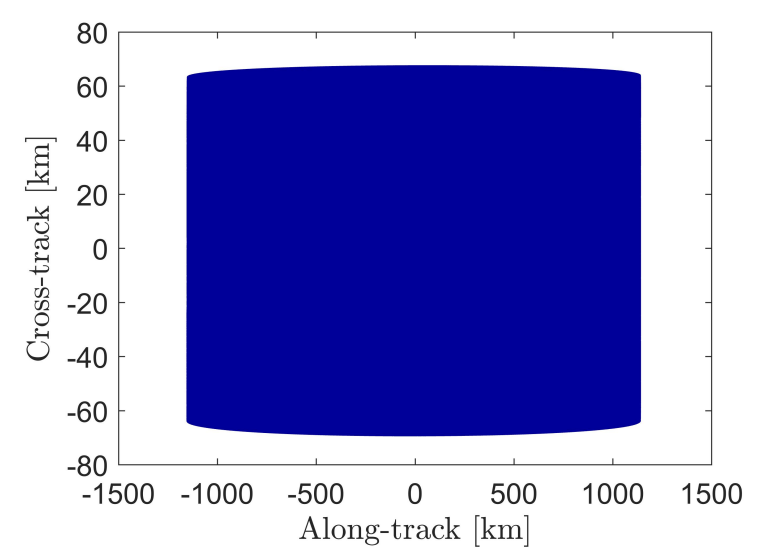

(c)

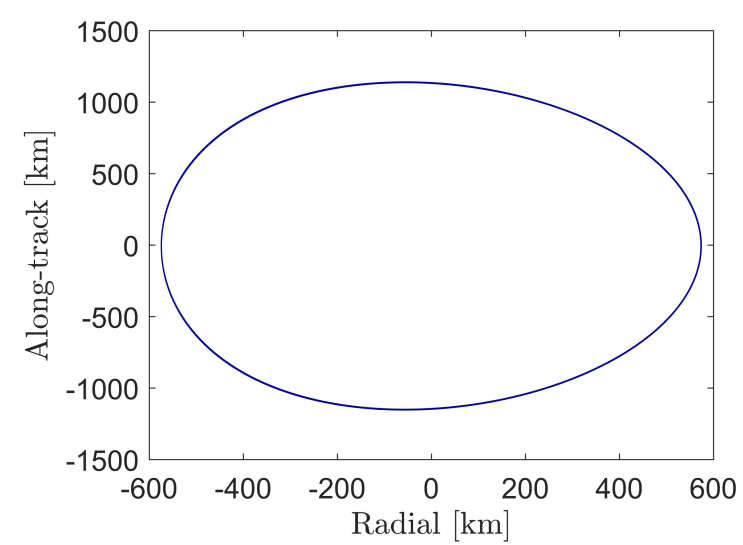

(b)

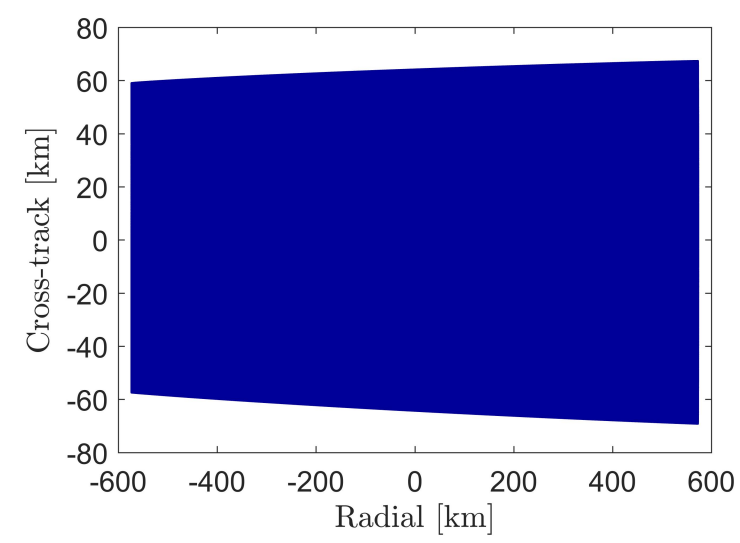

(d)

Fig. 12 Bounded relative orbits in the chief's local-vertical, local-horizontal coordinate frame. 


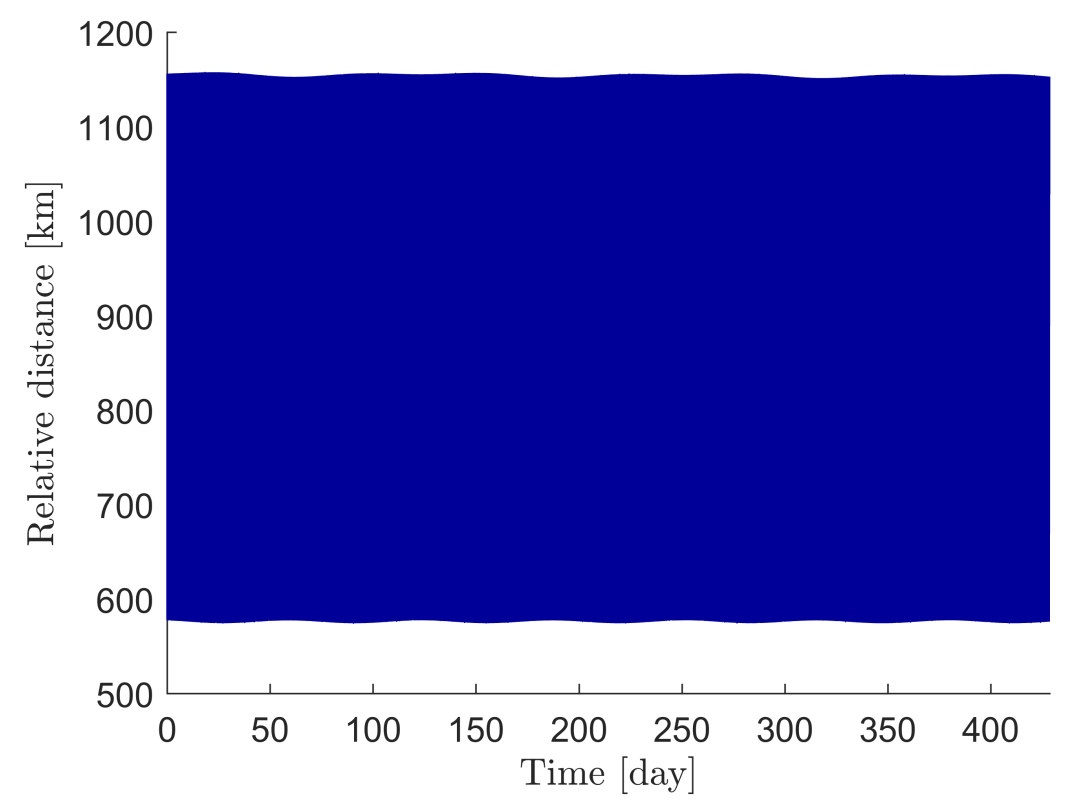

Fig. 13 Relative distance between the chief and deputy from the bounded relative motion.

to address this issue would be to apply the automatic domain splitting technique developed in [38] to guarantee that Taylor approximation of the Poincaré map is sufficiently accurate in the entire domain of interest. An alternative (and simpler) approach is proposed here by leveraging the fact that the solution computed with a single DA map provides a very good initial guess for a numerical refinement based on an optimization. The objective function to be minimized is

$$
J=\sigma\left(\rho_{p}\right)
$$

in which $\sigma(*)$ is the standard deviation and $\boldsymbol{\rho}_{p}$ is a vector containing the peaks of the relative distance during the propagation windows. The optimization variables are $\delta \dot{z}$ and $\delta \dot{\phi}$, whose first guess values are provided by the DA-based approach. The optimization is carried out using the Broyden-Fletcher-Goldfarb-Shanno algorithm implemented in the MATLAB fminunc function. Note that a single evaluation of Eq. (32) requires a fully numerical propagation of the equations of motion, resulting in numerically intensive computations. However, as the approach based on the high-order Poincaré map provides a very good initial condition, only a couple of iterations are required by the optimizer to converge. The results obtained with the numerical refinement are shown in the bottom panels of Fig. 18. Clearly, the drifts along both the radial and along-track directions is effectively suppressed and a more stable relative motion is achieved. Remarkably the correction applied by the optimizer to $[\delta \dot{z}, \delta \dot{\phi}]^{T}$ are just $\left[5.10759192655603 \times 10^{-7},-9.901059438085 \times 10^{-8}\right]^{T}$, showing the high sensitivity of the results to the initial conditions.

As a remark note that the objective function 32 is is selected among different options [e.g., $J=\left(\bar{T}_{d}-T_{d}{ }^{*}\right)^{2}+$ $\left.\left(\Delta \bar{\Omega}_{d}-\Delta \Omega_{d}^{*}\right)^{2}\right]$ as it provides accurate results with reduced computational time. 

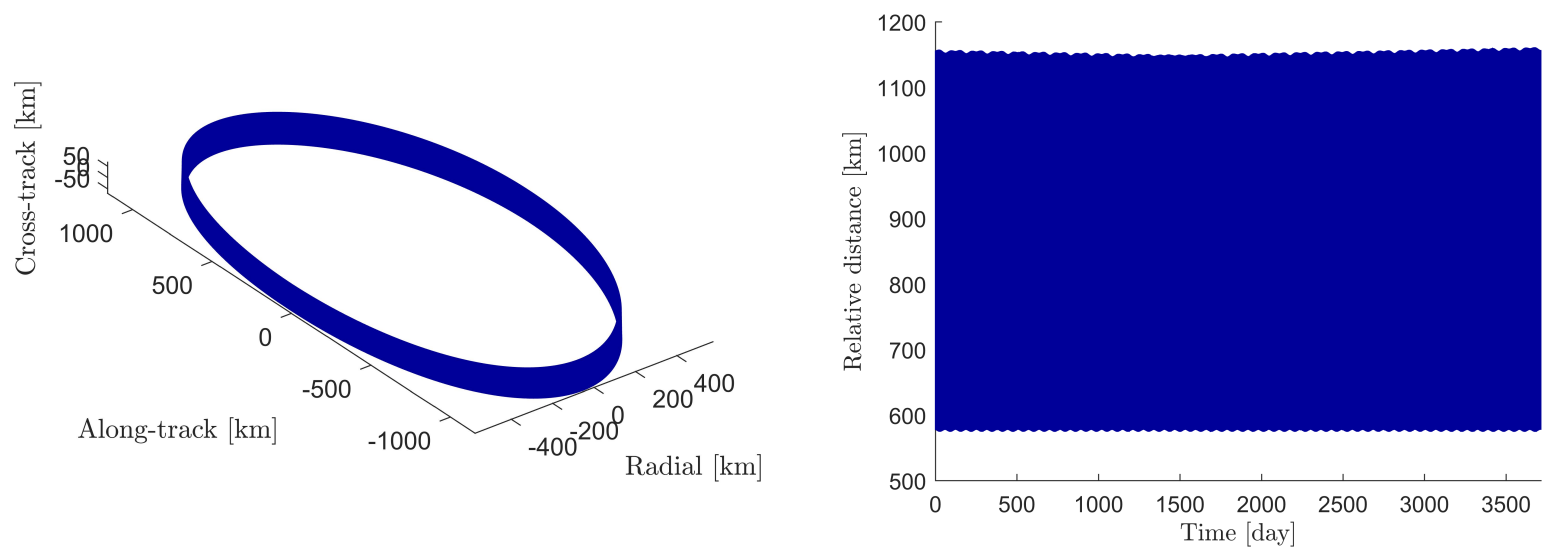

Fig. 14 Bounded relative orbit (left) and distance (right) for large time scale yielded by the same initial values in Fig. 12 ,
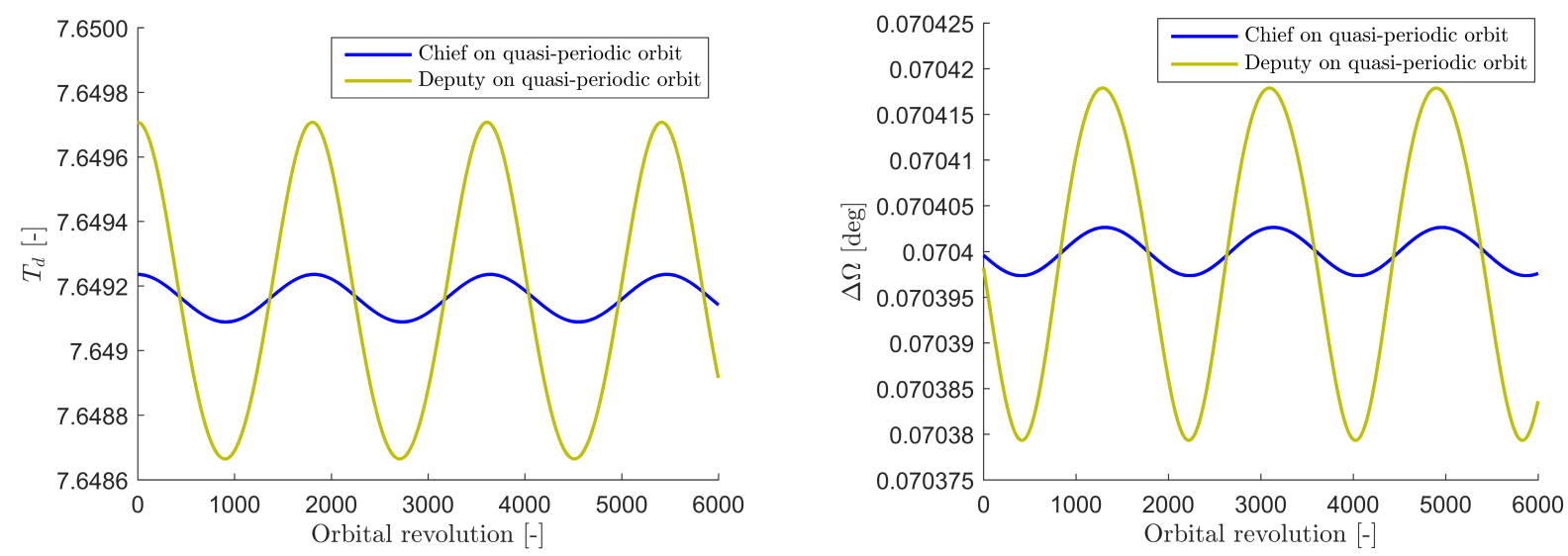

Fig. 15 Variation of $T_{d}$ (left) and $\Delta \Omega_{d}$ (right) for a chief and a deputy both initialized on quasi-periodic invariant curves with $\delta r=0.01$ and $\delta r=0.07$.
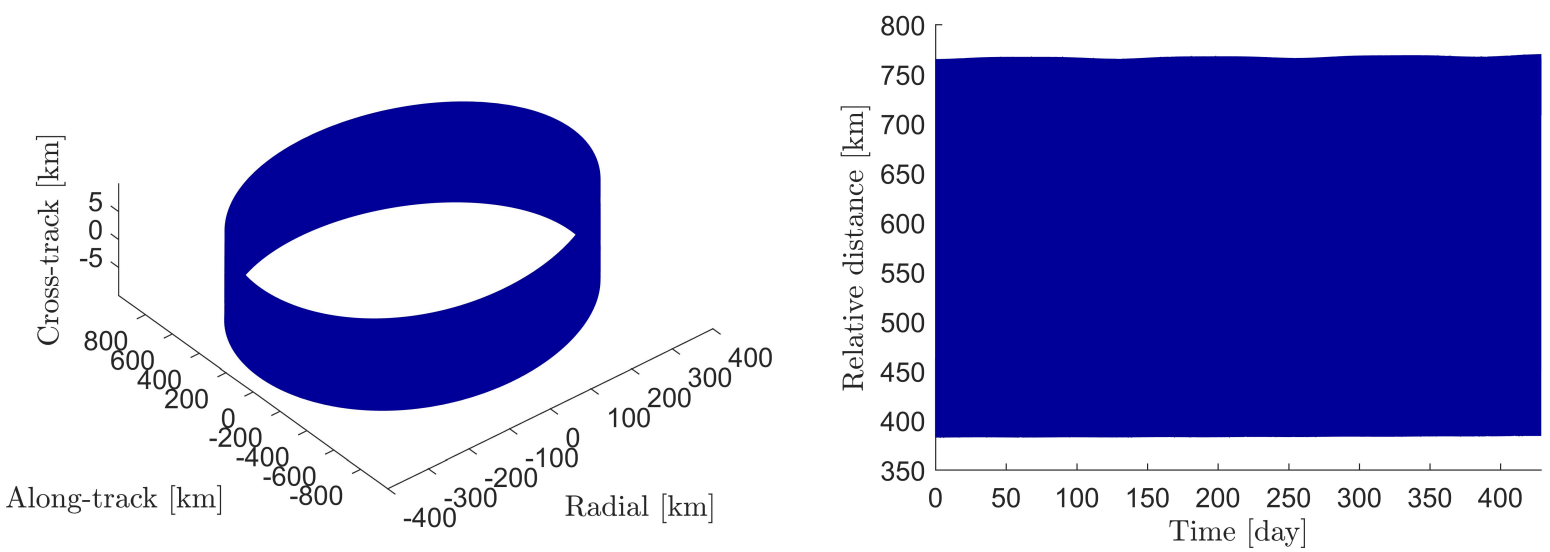

Fig. 16 Bounded relative motion (left) and corresponding relative distance (right) between a chief and a deputy both initialized on quasi-periodic invariant curves with $\delta r=0.01$ and $\delta r=0.07$. 


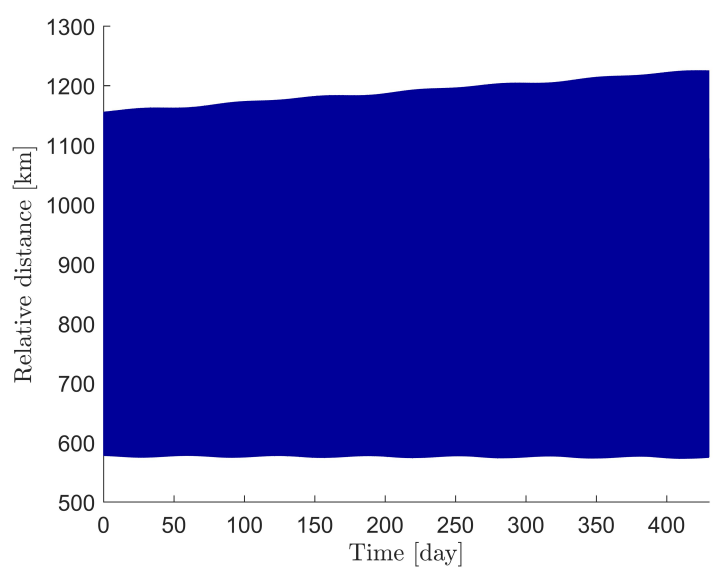

(a) case A, $\lambda_{0}=0 \mathrm{deg}$

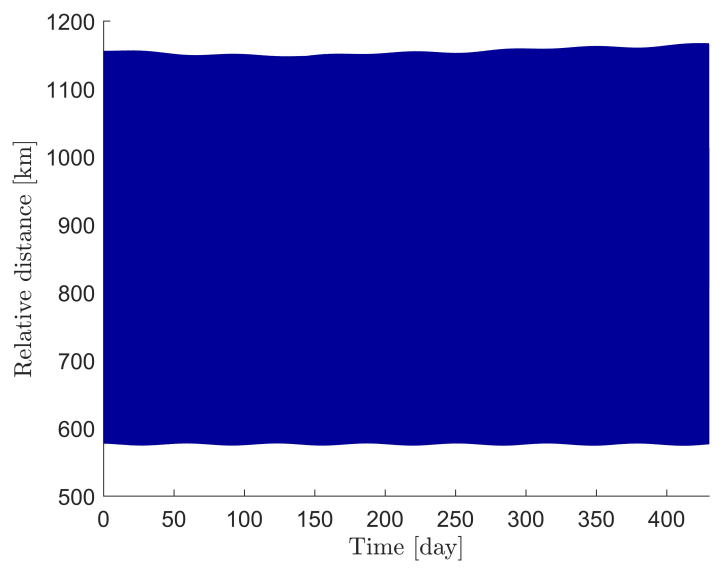

(c) case $\mathrm{A}, \lambda_{0}=10 \mathrm{deg}$

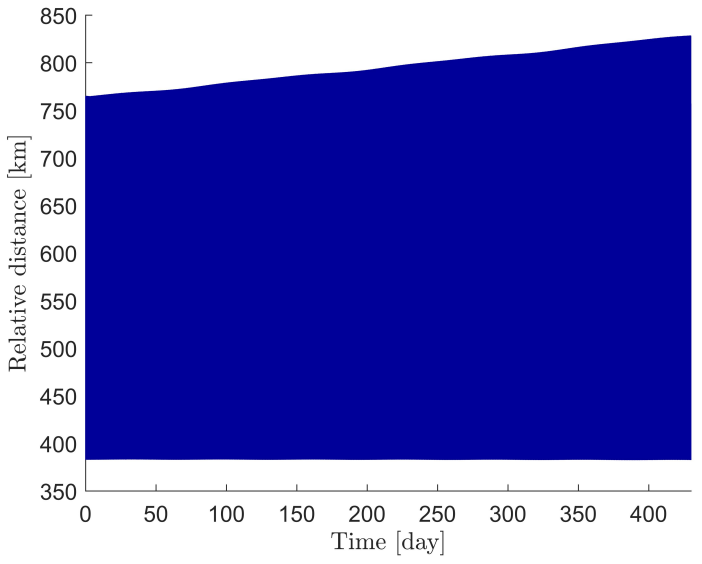

(b) case $\mathrm{B}, \lambda_{0}=0 \mathrm{deg}$

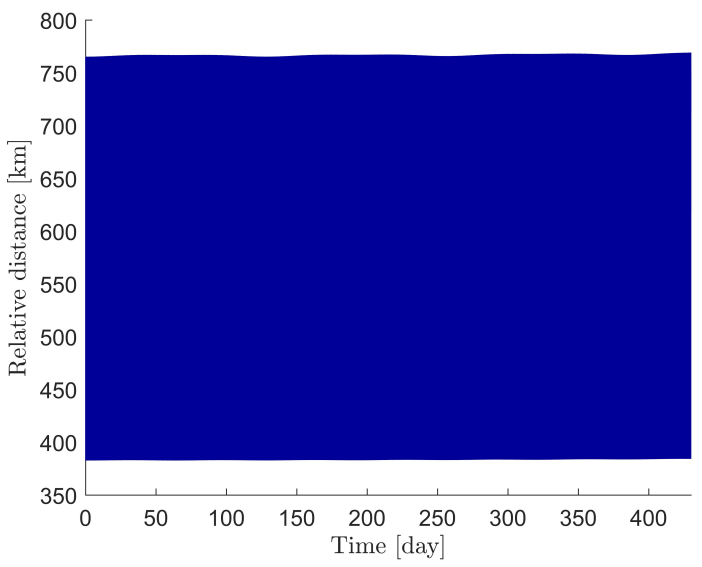

(d) case B, $\lambda_{0}=10 \mathrm{deg}$

Fig. 17 Relative distances of bounded initial conditions with respect to different initial longitudes: $\lambda_{0}=0 \mathrm{deg}$ (top); $\lambda_{0}=10 \mathrm{deg}$ (bottom) in the $J_{4,4}$ model. 

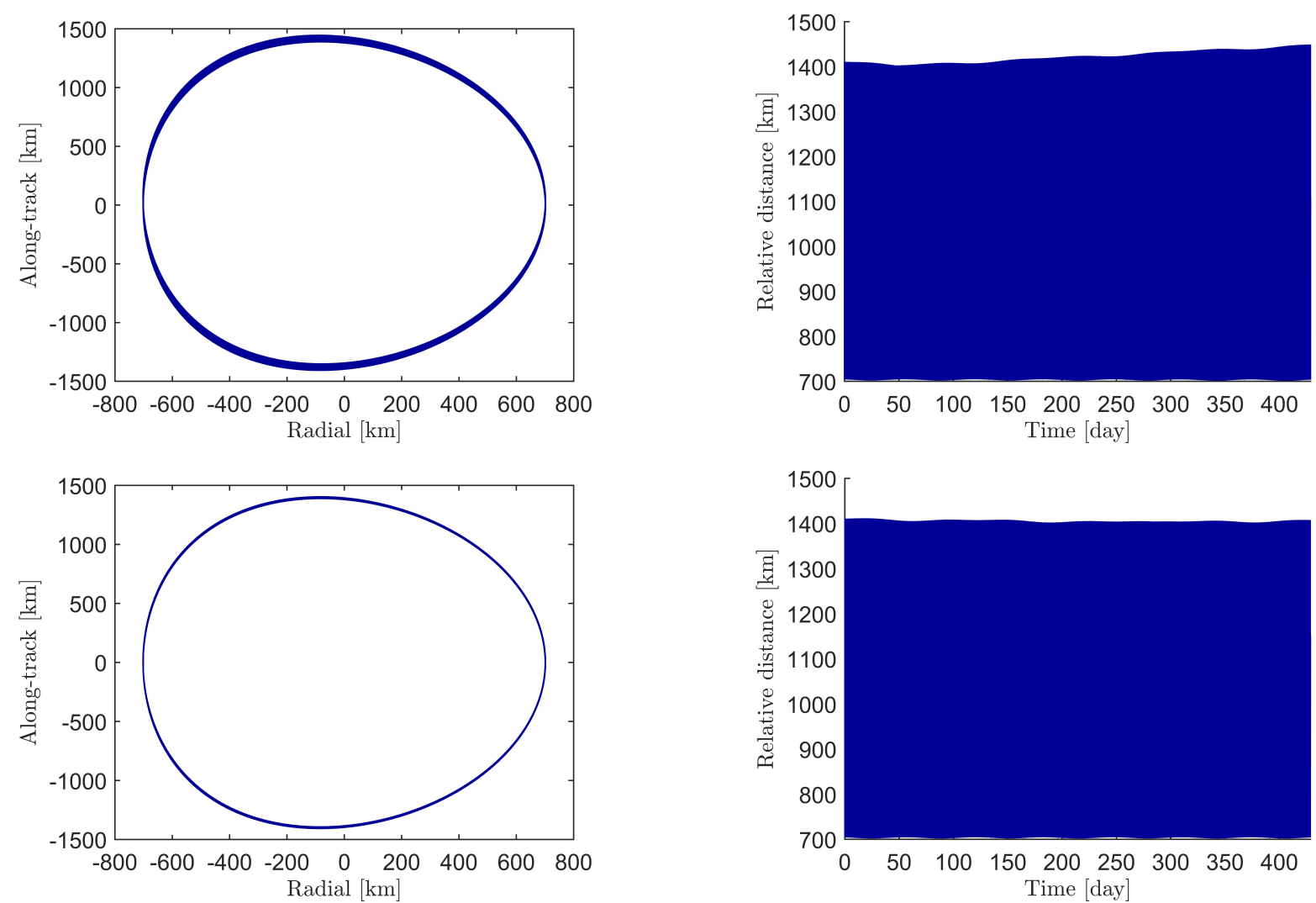

Fig. 18 Relative motion in the radial-along-track plane (left) and the relative distance (right) of relative orbits before (top) and after (bottom) the numerical refinement. 


\section{Bounded Relative Orbits Design via Optimization}

The algorithm for finding bounded relative motion presented in Sec. VI] is based on the numerical solution of two nonlinear equations, which allows for the determination of $\delta \dot{z}$ and $\delta \dot{\phi}$. The invariant curves are parametrized by $\delta r$ keeping fixed the value of $\delta \phi$. It is worth noting that in practical design problems, it may not be that necessary to compute the entire family of invariant curves, but just those initial conditions that provide a bounded relative motion with some specified properties. Moreover, the selection of the value of $\delta \phi$ can be relevant to ensure a desired shape for the relative geometry. In these cases the search for bounded relative motion can be better framed as an optimization problem, rather just than a constraint satisfaction one.

As explained in [40, 41], the radial and along-track motions share the same fundamental frequency and their bounds have a specific ratio value (radial:along-track $\approx 1: 2$ ), while in general the cross-track motion is independent from the in-plane motion. The dependency of the relative motion on the differential orbital elements of the chief and the deputy can be approximated by [41]

$$
\left\{\begin{array}{l}
r_{x}=\delta \rho, \\
r_{y}=\rho_{d}\left(\delta u+\delta \Omega \cos i_{c}\right), \\
r_{z}=\rho_{d}\left(-\sin i_{c} \delta \Omega \cos u_{d}+\delta i \sin u_{d}\right)=\rho_{d} \sqrt{\left(\sin i_{c} \delta \Omega\right)^{2}+(\delta i)^{2}} \sin \left(u_{d}+\beta\right),
\end{array}\right.
$$

where $r_{x}, r_{y}$, and $r_{z}$ are, respectively, the radial, along-track and cross-track relative positions and subscripts " $c$ " and " $d "$ denote the chief and the deputy. The orbit radius, argument of latitude, inclination of the spacecraft are represented by $\rho, u$, and $i ; \delta \rho, \delta u, \delta i$, and $\delta \Omega$ are the differential orbit radius, argument of latitude, inclination and RAAN between the chief and the deputy, respectively. The auxiliary variable $\beta$ satisfies $\tan \beta=-\sin i_{c} \delta \Omega / \delta i$.

Equation (33) provides the instantaneous variations of the radial, along-track, and cross-track separation that can be computed when the trajectories of both the chief and deputy are known. However, our approach to bounded relative orbit design is exclusively based on Poincaré sections, thus the orbital elements of the spacecraft are available only at equatorial crossings, which occur at different times. Nevertheless, with the assumption that within an orbital revolution the motion remains Keplerian (the usual approximation of orbital averaging), it is possible to fully reconstruct the relative motion between the chief and the deputy. To reduce the computational load we can reconstruct the relative motion at predefined times, e.g. when the chief crosses the equatorial plane (i.e., it is possible to reconstruct an approximation of the stroboscopic map). In order to do so the only part that needs some care is the computation of the term $\delta u$ in Eq. (33), which requires tracking the accumulated difference between the nodal periods of the chief and the deputy.

Figure 19 shows, in the left panels, the maximum values of the radial, along-track, and cross-track separation as functions of $\delta r$ and $\delta \phi$ reconstructed from the DA Poincaré map. These results are compared, in the right panels, with those computed by a fully numerical propagation of the bounded relative motion. It can be observed that the 

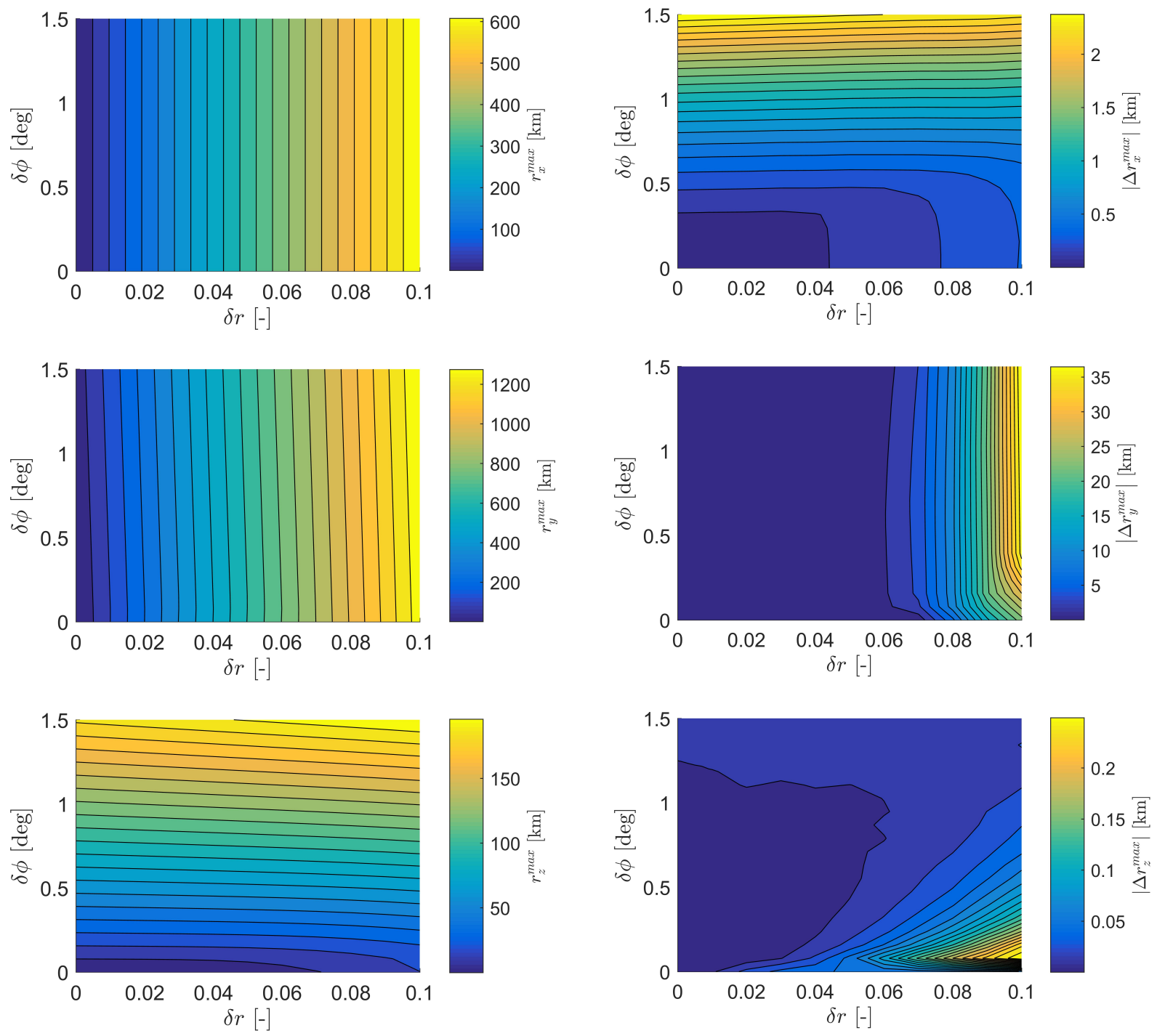

Fig. 19 Bounds of the bounded relative motions computed with the reconstruction (left) and the corresponding absolute errors compared with the actual values (right) as the function of $\delta r$ and $\delta \phi$. 

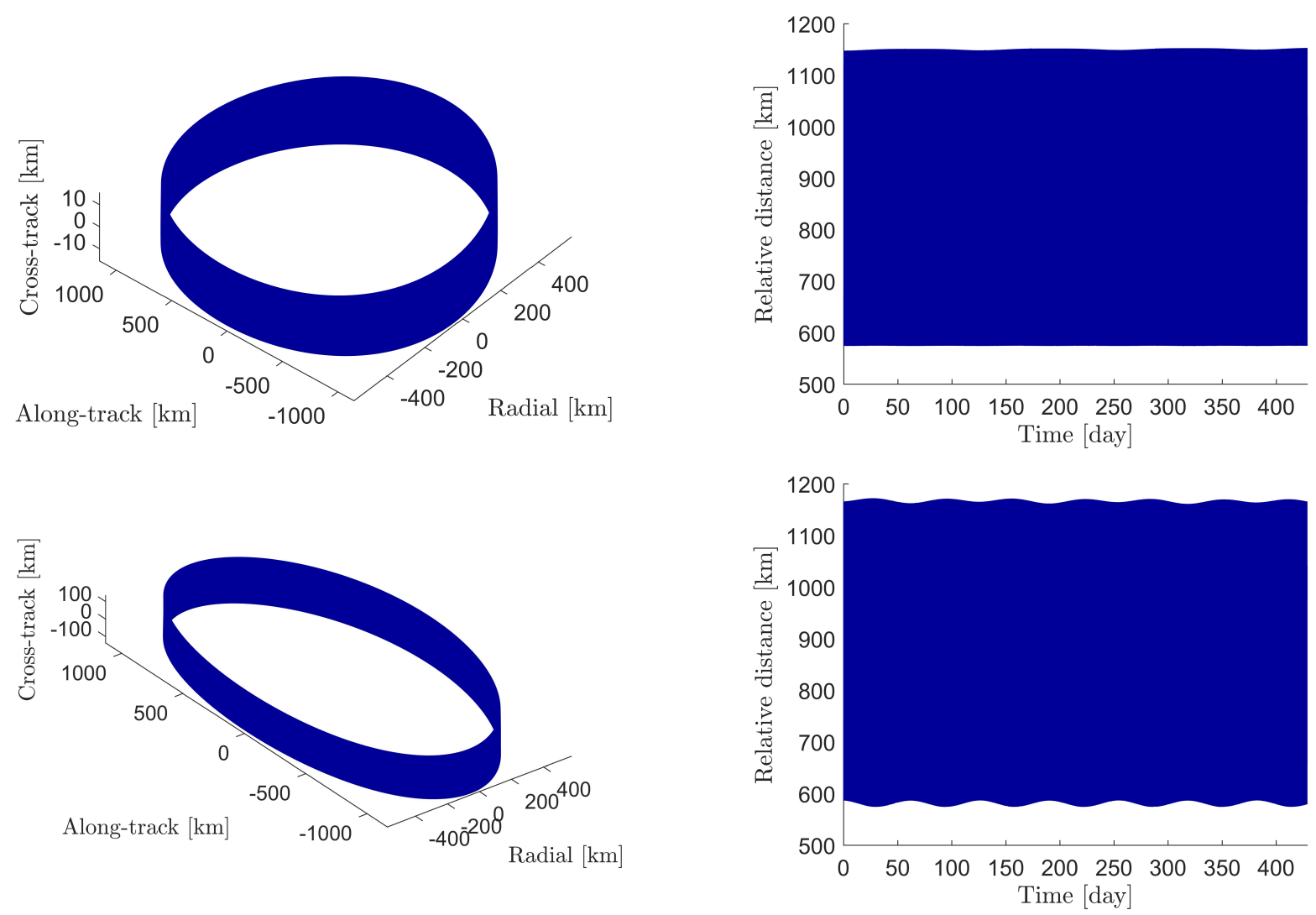

Fig. 20 The effect of initial longitudinal separation on the relative motion (left) and relative distance (right): $\delta \phi=0 \operatorname{deg}$ (top); $\delta \phi=1 \operatorname{deg}$ (bottom).

relevant features of the relative motion can be reconstructed quite accurately starting from the Poincaré maps from the point view of the size of relative geometry. The errors are induced due to both the accuracy of the Taylor expansion (for large deviation from the fixed point) and more importantly by the approximation introduced in the procedure of relative motion reconstruction (i.e., simplified formulas $(33)$ and Keplerian motion for $\delta u$ computation). Note that in case of a chief on a RGT-SS periodic orbit (i.e. almost polar orbit), $\delta r$ almost exclusively determines the radial and along-track motion, while $\delta \phi$ the cross-track separation. This last aspect is further analyzed in Fig.20, in which the chief is initialized on the RGT-SS periodic orbit of Table 1 and deputy on a quasi-periodic orbit with $\delta r=0.09$ and $\delta \phi$ equal to either $0 \mathrm{deg}$ or $1 \mathrm{deg}$. Comparing this figure with Fig. 12 (in which $\delta \phi=0.5 \mathrm{deg}$ ), it is apparent that the cross-track amplitude, and therefore the relative distances, increases with $\delta \phi$, and that "wave-like" fluctuations develop in the variation of relative distance. It needs to be stressed that the observation of these fluctuations is general and the initialization of SS (nearly polar) orbit would magnified it because of the term $\left|\sin i_{c} \delta \Omega\right|$ in Eq. 33].

Now that effects of both $\delta r$ and $\delta \phi$ on the relative motion have been addressed, we just provide an illustrative example on how the bounded relative motion design can be framed as an optimization problem. Consider the case in which we want to design a bounded relative geometry with prescribed targets on maximum radial and cross-track 
separations (along-track separation is not included in this example as it is coupled with the radial component). An optimization problem is formulated by defining the objective function

$$
J=\left(r_{x}^{\max }-r_{x}^{t}\right)^{2}+\left(r_{z}^{\max }-r_{z}^{t}\right)^{2}
$$

and the optimization vector $\boldsymbol{v}=[\delta r, \delta \phi]^{T}$, where $r_{x}^{t}$ and $r_{z}^{t}$ are, respectively, the target values of the maximum radial and cross-track separations prescribed by the mission designer. Note that for each value of the optimization vector the evaluation of the objective function requires solving for the bounded relative motion constraints, using the algorithm of Sec. VI/ The landscape of the objective function for $r_{x}^{t}=500 \mathrm{~km}$ and $r_{z}^{t}=120 \mathrm{~km}$ is shown in Fig.21, which is obtained with a $20 \times 20$ grid on the optimization variables. The initial guess for the optimization variables are

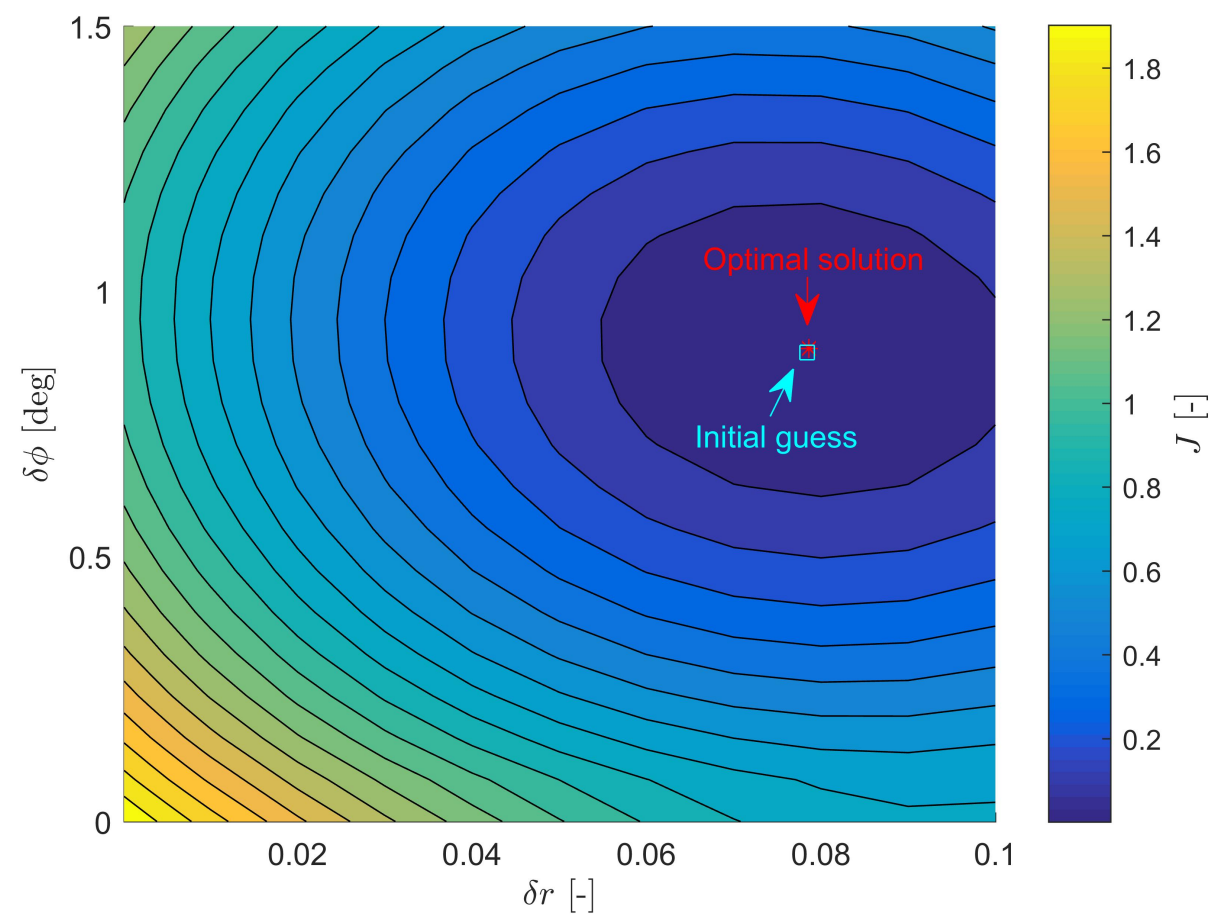

Fig. 21 Objective function evaluation with respect to $\delta r$ and $\delta \phi$.

derived from Eq. 33), resulting (in normalized units) in $\delta r=r_{x}^{t}=0.0783928$ and $\delta \phi=\frac{r_{z}^{t}}{\left(r_{c}+\delta r\right) \sin i_{c}}=0.0154398$, corresponding to the initial objective function $J=5.6383779 \times 10^{-5}$, as marked in Fig. 21 . In 8 iterations the MATLAB nonlinear solver fmincon (interior point algorithm) converges to the optimal solution $\delta r^{*}=0.0786168$ and $\delta \phi^{*}=0.0155731$, corresponding to $J^{*}=1.0004808 \times 10^{-5}$ as highlighted in Fig. 21. Finally, the optimal relative orbits satisfying both the prescribed boundaries and bounded constraints are provided in Fig.22. 


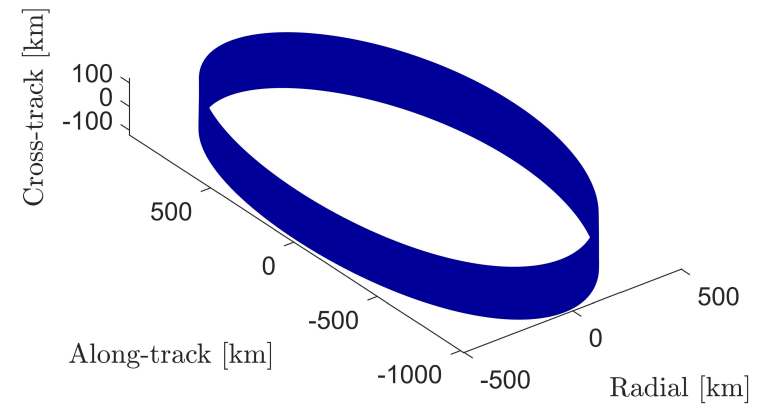

(a)

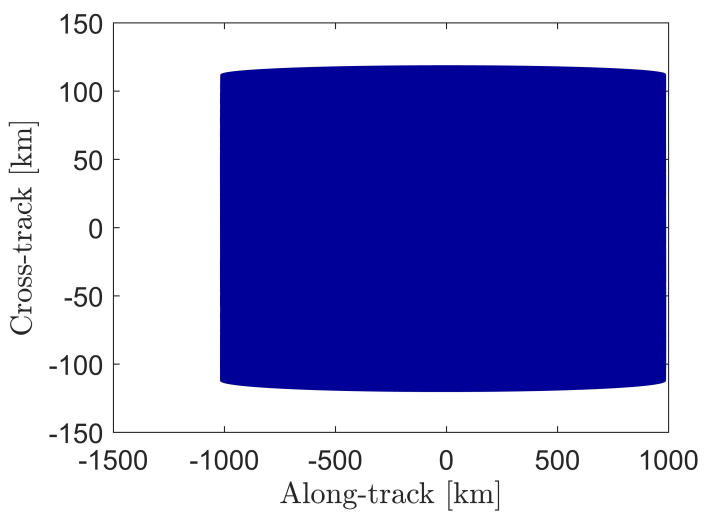

(c)

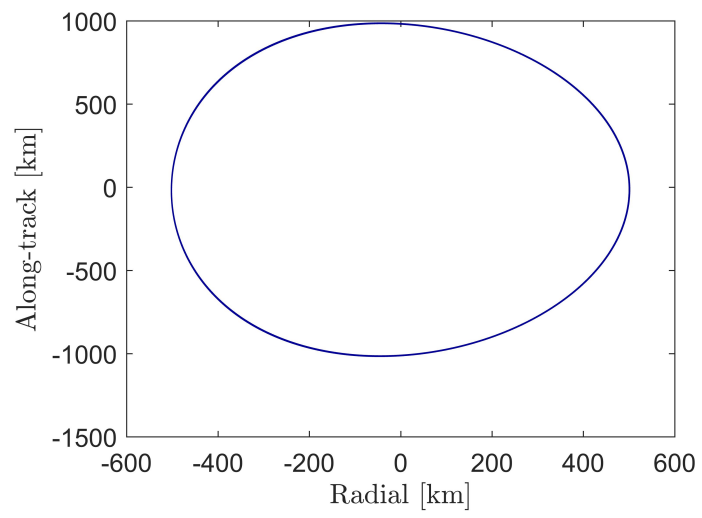

(b)

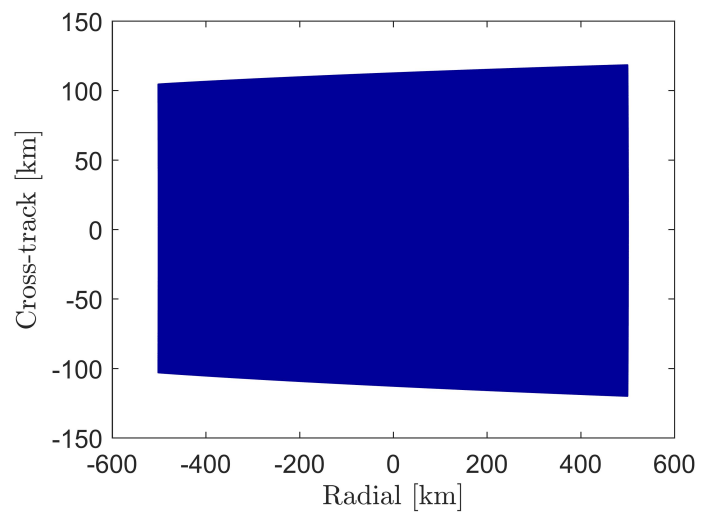

(d)

Fig. 22 Bounded relative orbits with the prescribed boundaries after the optimization. 


\section{Conclusions}

In this paper, the problem of generating naturally bounded relative orbits in the full zonal problem was addressed by combining Poincaré map method with high-order Taylor expansions. The main achievements of this work are summarized as two points: the computation of families of fixed points parametrized in the energy $(E)$ and the polar component of the angular momentum $\left(H_{z}\right)$, and the determination of invariant curves around them. Noticeably, both achievements were obtained by a single differential algebra (DA) propagation of the dynamics for a single nodal period.

It was shown that the computation of families of fixed points can be effectively used in mission design, e.g. by straightforwardly embedding repeat ground-track (RGT) and sun-synchronous (SS) constraints. Similarly, the determination of bounded relative motion conditions was reduced to a simple constraints satisfaction problem. This problem was solved numerically by exploiting the fact that the Taylor approximation of the Poincaré maps can be used to propagate points close to a periodic orbit for thousands of revolutions in a very efficient way. Although based on Taylor approximations, the proposed approach produces bounded relative motion with large apertures (up to over one thousand kilometers) and with negligible drifts in several years. This result is possible due to the fact that the proposed approach fully captures the nonlinearities of the problem and, like fully numerical approaches, it works directly with osculating quantities (thus, mean-to-osculating approximations are avoided). Although the accuracy of the Taylor approximations deteriorates when very large relative distances are considered, our approach provides very good initial conditions that are easily refined with a fully numerical techniques.

Current effort is focused on the application of normal form transformations to the Poincaré maps, which is expected to enable the evaluation of the average values of nodal period $\left(\bar{T}_{d}\right)$ and drift of right ascension of the ascending node per nodal period $\left(\bar{\Omega}_{d}\right)$ directly in the DA framework, thus reducing also the bounded relative orbits design to the solution of simple parametric implicit equations (PIEs).

\section{Acknowledgments}

This work was supported by the National Natural Science Foundation of China (11772024 and 11432001). Yanchao He acknowledges the funding from the Academic Excellence Foundation of BUAA for PhD students, as well as the helpful discussions with Juan Félix San Juan, David Gondelach, Laura Pirovano, and Gennaro Principe during his research periods spent at the Universidad de La Rioja. Ming Xu acknowledges the financial supports from Aerospace

Science Foundation by China Aerospace Science and Industry Corporation, and Shanghai Space Science and Technology Innovation Foundation (SAST2017-033), and the Fundamental Research Funds for the Central Universities.

\section{References}

[1] D’Amico, S., Ardaens, J. S., and Larsson, R., "Spaceborne Autonomous Formation Flying Experiment on the PRISMA Mission," Journal of Guidance, Control and Dynamics, Vol. 35, No. 3, 2012, pp. 834-850. doi:10.2514/1.55638. 
[2] Montenbruck, O., Kirschner, M., D’Amico, S., and Bettadpur, S., "E/I-Vector Separation for Safe Switching of the GRACE Formation," Aerospace Science and Technology, Vol. 10, No. 7, 2006, pp. 628-635. doi:10.1016/j.ast.2006.04.001.

[3] D’Amico, S., and Montenbruck, O., "Proximity Operations of Formation-Flying Spacecraft Using an Eccentricity/Inclination Vector Separation," Journal of Guidance, Control, and Dynamics, Vol. 29, No. 3, 2005, pp. 554-563. doi:10.2514/1.15114.

[4] Clohessy, W. H., and Wiltshire, R. S., “Terminal Guidance System for Satellite Rendezvous,” Journal of the Aerospace Sciences, Vol. 27, No. 9, 1960, pp. 653-658. doi:10.2514/8.8704.

[5] Sullivan, J., Grimberg, S., and D’Amico, S., "Comprehensive Survey and Assessment of Spacecraft Relative Motion Dynamics Models," Journal of Guidance, Control, and Dynamics, Vol. 40, No. 8, 2017, pp. 1837-1859. doi:10.2514/1.G002309.

[6] Inalhan, G., Tillerson, M., and How, J. P., "Relative Dynamics and Control of Spacecraft Formations in Eccentric Orbits," Journal of Guidance, Control, and Dynamics, Vol. 25, No. 1, 2002, pp. 48-59. doi:10.2514/2.4874.

[7] Gurfil, P., "Relative Motion Between Elliptic Orbits: Generalized Boundedness Conditions and Optimal Formationkeeping," Journal of Guidance, Control, and Dynamics, Vol. 28, No. 4, 2012, pp. 761-767. doi:10.2514/1.9439.

[8] Vadali, S., Schaub, H., and Alfriend, K., "Initial Conditions and Fuel-optimal Control for Formation Flying of Satellites," AIAA Guidance, Navigation and Control Conference, AIAA, Portland, OR, 1999.

[9] Alfriend, K. T., Vadali, S. R., and Schaub, H., "Formation Flying Satellites: Control By an Astrodynamicist," Celestial Mechanics and Dynamical Astronomy, Vol. 81, No. 1-2, 2001, pp. 57-62. doi:10.1023/A:101330710.

[10] Breger, L., and How, J. P., "Gauss's Variational Equation-Based Dynamics and Control for Formation Flying Spacecraft," Journal of Guidance, Control, and Dynamics, Vol. 30, No. 2, 2007, pp. 437-448. doi:10.2514/1.22649.

[11] Frey, G. R., Petersen, C. D., Leve, F. A., Kolmanovsky, I. V., and Girard, A. R., "Constrained Spacecraft Relative Motion Planning Exploiting Natural Motion Trajectories and Invariance," Journal of Guidance, Control, and Dynamics, Vol. 40, No. 12, 2017, pp. 3100-3115. doi:10.2514/1.G002914.

[12] Schaub, H., and Alfriend, K. T., “ $J_{2}$ Invariant Relative Orbits for Spacecraft Formations,” Celestial Mechanics and Dynamical Astronomy, Vol. 79, No. 2, 2001, pp. 77-95. doi:10.1023/A:1011161811472.

[13] Gurfil, P., “Generalized Solutions for Relative Spacecraft Orbits Under Arbitrary Perturbations,” Acta Astronautica, Vol. 60, No. 2, 2007, pp. 61-78. doi:10.1016/j.actaastro.2006.07.013.

[14] Dang, Z., Wang, Z., and Zhang, Y., "Improved Initialization Conditions and Single Impulsive Maneuvers for $J_{2}$-Invariant Relative Orbits," Celestial Mechanics and Dynamical Astronomy, Vol. 121, No. 3, 2015, pp. 301-327. doi:10.1007/s10569-014-9601-4.

[15] He, Y., and Xu, M., "Bounded Relative Orbits in the Zonal Gravitational Field for Formation Flying," Journal of Guidance, Control, and Dynamics, Published online, March 26, 2018. doi:10.2514/1.G003107. 
[16] Lara, M., and Gurfil, P., "Integrable Approximation of $J_{2}$-Perturbed Relative Orbits," Celestial Mechanics and Dynamical Astronomy, Vol. 114, No. 3, 2012, pp. 229-254. doi:10.1007/s10569-012-9437-8.

[17] Chu, J., Guo, J., and Gill, E. K. A., "Long-term Passive Distance-Bounded Relative Motion in the Presence of $J_{2}$ Perturbations," Celestial Mechanics and Dynamical Astronomy, Vol. 121, No. 4, 2015, pp. 385-413. doi:10.1007/s10569-015-9603-x.

[18] Martinuşi, V., and Gurfil, P., "Solutions and Periodicity of Satellite Relative Motion Under Even Zonal Harmonics Perturbations," Celestial Mechanics and Dynamical Astronomy, Vol. 111, No. 4, 2011, pp. 387-414. doi:10.1007/s10569-011-9376-9.

[19] Martinusi, V., and Gurfil, P., “Analytical Derivation of Single-Impulse Maneuvers Guaranteeing Bounded Relative Motion Under $J_{2}$, "Journal of Guidance, Control, and Dynamics, Vol. 37, No. 1, 2014, pp. 233-242. doi:10.2514/1.60238.

[20] Koon, W. S., Marsden, J. E., Murray, R. M., and Masdemont, J., “ $J_{2}$ Dynamics and Formation Flight,” AIAA Guidance, Navigation, and Control Conference and Exhibit, AIAA, Montreal, Canada, 2001.

[21] Broucke, R. A., "Numerical Integration of Periodic Orbits in the Main Problem of Artificial Satellite Theory," Celestial Mechanics and Dynamical Astronomy, Vol. 58, No. 2, 1994, pp. 99-123. doi:10.1007/BF00695787.

[22] Xu, M., Wang, Y., and Xu, S., "On the Existence of $J_{2}$ Invariant Relative Orbits from the Dynamical System Point of View," Celestial Mechanics and Dynamical Astronomy, Vol. 112, No. 4, 2012, pp. 427-444. doi:10.1007/s10569-012-9401-7.

[23] Baresi, N., and Scheeres, D. J., "Bounded Relative Motion Under Zonal Harmonics Perturbations," Celestial Mechanics and Dynamical Astronomy, Vol. 127, No. 4, 2017, pp. 527-548. doi:10.1007/s10569-016-9737-5.

[24] Baresi, N., and Scheeres, D. J., "Design of Bounded Relative Trajectories in the Earth Zonal Problem," Journal of Guidance, Control, and Dynamics, Vol. 40, No. 12, 2017, pp. 3075-3087. doi:10.2514/1.G002603.

[25] Baresi, N., Olikara1, Z. P., and Scheeres, D. J., "Fully Numerical Methods for Continuing Families of Quasi-Periodic Invariant Tori in Astrodynamics," The Journal of the Astronautical Sciences, Published online, January 16, 2018. doi: 10.1007/s40295-017-0124-6.

[26] Wittig, A., and Armellin, R., "High Order Transfer Maps for Perturbed Keplerian Motion,” Celestial Mechanics and Dynamical Astronomy, Vol. 122, No. 4, 2015, pp. 333-358. doi:10.1007/s10569-015-9621-8.

[27] Berz, M., Modern Map Methods in Particle Beam Physics, Academic Press, London, 1999, pp. 81-117.

[28] Armellin, R., Lizia, P. D., Bernelli-Zazzera, F., Berz, M., and Di Lizia, P., “Asteroid Close Encounters Characterization Using Differential Algebra: The Case of Apophis," Celestial Mechanics and Dynamical Astronomy, Vol. 107, No. 4, 2010, pp. 451-470. doi:10.1007/s10569-010-9283-5.

[29] Valli, M., Armellin, R., Lizia, P. D., and Lavagna, M. R., "Nonlinear Mapping of Uncertainties in Celestial Mechanics,” Journal of Guidance, Control, and Dynamics, Vol. 36, No. 1, 2013, pp. 48-63. doi:10.2514/1.58068. 
[30] Morselli, A., Armellin, R., Lizia, P. D., and Zazzera, F. B., "A High Order Method for Orbital Conjunctions Analysis: Monte Carlo Collision Probability Computation," Advances in Space Research, Vol. 55, No. 1, 2015, pp. 311-333. doi: 10.1016/j.asr.2014.09.003.

[31] Wittig, A., Colombo, C., and Armellin, R., "Long-Term Density Evolution Through Semi-Analytical and Differential Algebra Techniques," Celestial Mechanics and Dynamical Astronomy, Vol. 128, No. 4, 2017, pp. 435-452. doi:10.1007/s10569-0179756-x.

[32] Armellin, R., and Lizia, P. D., "Probabilistic Optical and Radar Initial Orbit Determination," Journal of Guidance, Control, and Dynamics, Vol. 41, No. 1, 2018, pp. 101-118. doi:10.2514/1.G002217.

[33] He, Y., Xu, M., Jia, X., and Armellin, R., "High-Precision Repeat-Groundtrack Orbit Design and Maintenance for Earth Observation Missions," Celestial Mechanics and Dynamical Astronomy, Vol. 128, No. 2-3, 2017, pp. 275-294. doi: 10.1007/s10569-017-9753-0.

[34] Lara, M., "Searching for Repeating Ground Track Orbits: A Systematic Approach," The Journal of the Astronautical Sciences, Vol. 47, No. 3-4, 1999, pp. 177-188.

[35] Rasotto, M., Morselli, A., Wittig, A., Massari, M., Di Lizia, P., Armellin, R., Valles, C., and Ortega, G., "Differential Algebra Space Toolbox for Nonlinear Uncertainty Propagation in Space Dynamics," 6th International Conference on Astrodynamics Tools and Techniques, ICATT 2016, Darmstadt, Germany, 2016, pp. 1-11.

[36] Lizia, P. D., Armellin, R., and Lavagna, M., “Application of High Order Expansions of Two-Point Boundary Value Problems to Astrodynamics," Celestial Mechanics and Dynamical Astronomy, Vol. 102, No. 4, 2008, pp. 355-375. doi: 10.1007/s10569-008-9170-5.

[37] Cook, G. E., "Luni-Solar Perturbations of the Orbit of an Earth Satellite," Geophysical Journal of the Royal Astronomical Society, Vol. 6, No. 3, 1962, pp. 271-291. doi:10.1111/j.1365-246X.1962.tb00351.x.

[38] Wittig, A., Di Lizia, P., Armellin, R., Makino, K., Bernelli-Zazzera, F., and Berz, M., "Propagation of Large Uncertainty Sets in Orbital Dynamics by Automatic Domain Splitting," Celestial Mechanics and Dynamical Astronomy, Vol. 122, No. 3, 2015, pp. 239-261. doi:10.1007/s10569-015-9618-3.

[39] Armellin, R., Wittig, A., and San Juan, J. F., "High Order Transfer Map Method and General Perturbation Techniques Applied to Perturbed Keplerian Motion,” Astrodynamics 2015, Vol. 156, American Astronautical Society, 2016, pp. 1067-1084.

[40] Vadali, S. R., "An Analytical Solution for Relative Motion of Satellites," Dynamics and Control of Systems and Structures in Space 2002, Cranfield University, Cranfield, UK, July 2002, pp. 309-316.

[41] Alfriend, K. T., Vadali, S. R., Gurfil, P., How, J. P., and Breger, L. S., Spacecraft Formation Flying: Dynamics, Control and Navigation, Batterworth-Heinemann, Oxford, 2010, pp. 144-146. 